\title{
Evaluation of sulfane sulfur bioeffects via a mitochondria-targeting selenium-containing near-infrared fluorescent probe
}

\author{
Min Gao ${ }^{\text {a, b }}$, Rui Wang ${ }^{\text {a }}$, Fabiao Yu ${ }^{\text {a, }}{ }^{* *}$, Lingxin Chen ${ }^{\text {a, * }}$ \\ ${ }^{a}$ Key Laboratory of Coastal Environmental Processes and Ecological Remediation, Research Centre for Coastal Environmental Engineering and Technology, \\ Yantai Institute of Coastal Zone Research, Chinese Academy of Sciences, Yantai, 264003, China \\ ${ }^{\mathrm{b}}$ University of Chinese Academy of Sciences, Beijing, 100049, China
}

\section{A R T I C L E I N F O}

Article history:

Received 26 November 2017

Received in revised form

27 December 2017

Accepted 6 January 2018

Available online 11 January 2018

\section{Keywords:}

Fluorescent probes

Sulfane sulfur

Mitochondria-targeting

In vivo bioimaging

Hypoxia stress

\begin{abstract}
A B S T R A C T
As a crucial member in antioxidant regulatory systems, sulfane sulfur plays essential roles in cytoprotective mechanisms by directly eliminating ROS and altering ROS-mediated redox signaling. Despite the rising interests in sulfane sulfur, there only a few bio-compatible methods are available for its direct detection. Moreover, most of the existing methods cannot meet the requirements of real-time detection due to the reactive and labile chemical properties of sulfane sulfur. Therefore, we strive to clarify the mutual relationship between mitochondria sulfane sulfur and ROS under hypoxia stress. Herein, we report a near-infrared fluorescent probe $\mathrm{Mito}-\mathrm{SeH}$ for the selective imaging of mitochondrial sulfane sulfur in cells and in vivo under hypoxia stress. Mito-SeH includes three moieties: a selenol group ( $-\mathrm{SeH}$ ) as the stronger sulfur-acceptor; a near-infrared azo-BODIPY fluorophore as the fluorescent modulator; a lipophilic alkyltriphenylphosphonium cation as the mitochondrial delivery. Mito-SeH exhibits excellent selectivity and sensitivity towards the detection of mitochondria sulfane sulfur. The hypoxia response behavior of Mito-SeH is evaluated in monolayer cell and three-dimensional multicellular spheroid to clarify the relationship between sulfane sulfur and hypoxia. We confirm that sulfane sulfur protection mechanism against hypoxia is to inhibition of caspase-dependent apoptosis through directly scavenging ROS pathway. The probe is also applied to measurement of sulfane sulfur in ex vivo-dissected organs of hypoxic mouse model, as well as the probe is successfully used for real-time monitoring the changes of sulfane sulfur and ROS in acute ischemia mice model. We suggest that sulfane sulfur may be a novel therapeutic agent for hypoxia-induced injury.
\end{abstract}

(C) 2018 Elsevier Ltd. All rights reserved.

\section{Introduction}

Sulfane sulfur is a class of sulfur-containing small biomolecules including a highly reactive sulfur atom covalently bound to another sulfur atom $\left(S^{0}\right)$ [1]. It is generally presented in forms of persulfides (RSSH), polysulfides ( $\left.R-S_{n}-R, n \geq 3\right)$, hydrogen polysulfides $\left(\mathrm{H}_{2} \mathrm{~S}_{\mathrm{n}}\right.$, $\mathrm{n} \geq 2$ ) and elemental sulfur $\left(\mathrm{S}_{8}\right)$ simultaneously accompanying with their thiosulfoxide tautomers. Sulfane sulfur assumes pivotal roles in balancing the redox of intracellular milieu, regulating the activities of enzymes, synthesizing cofactors, and modulating sulfuration of tRNA [2]. Sulfane sulfur can be endogenously produced by enzymes, such as cystathionine $\gamma$-lyase (CSE) [3,4], cystathionine $\beta$ -

\footnotetext{
* Corresponding author.

** Corresponding author.

E-mail addresses: fbyu@yic.ac.cn (F. Yu), lxchen@yic.ac.cn (L. Chen).
}

synthase (CBS) [5], as well as 3-mercaptopyruvate sulfurtransferase (3-MST) [6], which are considered to be involved in the formation of hydrogen sulfide $\left(\mathrm{H}_{2} \mathrm{~S}\right)$ for a long time. Some evidences reveal that sulfane sulfur may be the physiologically relevant pool of $\mathrm{H}_{2} \mathrm{~S}$ for its crucial participation in cell regulation processes through activation or inactivation of enzymes [7], or $\mathrm{H}_{2} \mathrm{~S}$ is merely an ultimate product of sulfane sulfur, which is released when the biological events are activated [8-11]. That is, sulfane sulfur and $\mathrm{H}_{2} \mathrm{~S}$ always coexist in biological systems. Given the special relationship between them, the increasing suggestions reveal that sulfane sulfur may be the actual signaling molecules instead of $\mathrm{H}_{2} \mathrm{~S}$ [12].

Hypoxia stress can induce the overproduction of reactive oxygen species (ROS) [13-17] which is a major cause of cellular damage and death $[18,19]$. However, cells can maintain the intracellular redox homeostasis through its intrinsic antioxidant regulatory systems built by the evolution of cells. Sulfane sulfur is one of the crucial contributors of antioxidant defense systems in cells [4]. 
These sulfur-containing species can ameliorate oxidative stress and prevent cells from irreversible damage. Although the increasing level of 3-MST for sulfane sulfur generation under hypoxia stress in cells has been suggested $[10,20]$, the direct relationship between hypoxia and sulfane sulfur is far from fully illustrated [21]. We hypothesize that the phenomenon of intracellular sulfane sulfur changes is host defense for the cell self-protection under hypoxia stress. Therefore, in this work, we strive to clarify the relationship between sulfane sulfur and hypoxia for further understanding the physiological and pathological roles of sulfane sulfur in living cells.

The thiosulfoxide tautomers of sulfane sulfur contains a labile and highly reactive sulfur atom covalently bound to another sulfur atom at a reduced oxidation state 0 or -1 [2]. Due to this particular feature, the very sulfur atom readily leaves the compound structure and can be transferred to nucleophilic acceptors such as cyanide $\left(\mathrm{CN}^{-}\right)$. The general method for sulfane sulfur detection is based on the reaction with $\mathrm{CN}^{-}$to yield thiocyanate which is easily measured as ferric thiocyanate (Scheme 1) [22]. In addition, other methods, such as ion chromatography, gas chromatography and high performance liquid chromatography (HPLC), were also used to detect sulfane sulfur [23]. But these methods typically required sample pretreatment, which limited their applications for the real-time and in situ detection of sulfane sulfur in biological samples. Moreover, the catabolism of sulfane sulfur is so fast as to result in continuous fluctuation in its concentration. Some biothiols, such as glutathione which presents at millimolar levels in cells, can often interfere the detection of sulfane sulfur. Therefore, it is necessary to develop fast, accurate, and real-time approaches for sulfane sulfur detection in cells and in vivo. Compared with other bio-analytical methods, fluorescence imaging offers high sensitivity, real-time imaging, and high spatiotemporal resolution. Therefore, small molecule-based fluorescent probes have become indispensable chemical tools for the detection of reactive small molecules in cells and in vivo [24-29]. There have been many efforts to achieve elegant fluorescent probes for intracellular RSS detection. Unfortunately, few sulfane sulfur-sensitive probes have been reported so far [30-34]. Most of the current fluorescent probes are designed for the selective detection of a single subclass of sulfane sulfur, such as $\mathrm{H}_{2} \mathrm{~S}_{\mathrm{n}}$ and hydropersulfide (Cys-SSH) [3,5,35-41]. However, it is important to define the changes of the overall sulfane sulfur in cells, because the total level of intracellular sulfane sulfur may indicate the health of the living body. Therefore, the new fluorescent probe for the whole sulfane sulfur detection is still urgent need [30]. Furthermore, fluorescent probes whose excitation and emission locate in NIR region allow for in vivo imaging with high tissue penetration, minimal photo damage to biological samples, and minimum interference from background autofluorescence in living systems. Mitochondria are a prominent site for ROS production. Despite the high antioxidative capacity of mitochondria, the excessive ROS release can cause a variety of physiological dysfunction. Since studies have implied that sulfane sulfur is associated with redox homeostasis in mitochondria, a fluorescent probe that is able to provide information on sulfane sulfur in mitochondria is especially appreciated. To develop a new NIR fluorescent probe for rapid and selective imaging mitochondrial sulfane sulfur in cells and in vivo becomes our target.

Herein, we present a NIR fluorescent probe, Mito-SeH, which possesses enhanced sensitivity that allows for preferential imaging of mitochondrial sulfane sulfur in living cells and in vivo. The probe includes a sulfane sulfur response moiety 2-hydroselenobenzoate fragment, a mitochondria-targeted unit lipophilic triphenylphosphonium cation and an NIR fluorophore BODIPY. The probe can also be applied to evaluate the mutual relationship between sulfane sulfur and ROS in monolayer cells under hypoxic condition, in three-dimensional multicellular spheroid, in ex vivo-dissected organs of hypoxic mice model, and in acute ischemia mice model, which can further explain the protective effects of sulfane sulfur through inhibiting mitochondrial caspase-dependent apoptosis under hypoxia stress.

\section{Experimental}

\subsection{Synthesis and characterization of Mito-SeH}

\subsubsection{Synthesis of $\mathrm{Na}_{2} \mathrm{Se}_{2}$}

Selenium ( $5.13 \mathrm{~g}, 0.065 \mathrm{mmol})$ and sodium borohydride $(4.45 \mathrm{~g}$, $0.13 \mathrm{mmol}$ ) were added to $\mathrm{H}_{2} \mathrm{O}(35 \mathrm{~mL})$ and the mixture were stirred for $30 \mathrm{~min}$ under nitrogen atmosphere, then selenium ( $5.13 \mathrm{~g}, 0.065 \mathrm{mmol}$ ) was added to the mixture and then stirred for another $30 \mathrm{~min}$ under nitrogen atmosphere to produce a brown red solution. Then the solution was alkalization with $25 \mathrm{~mL}$ sodium hydroxide $(10 \mathrm{M})$.

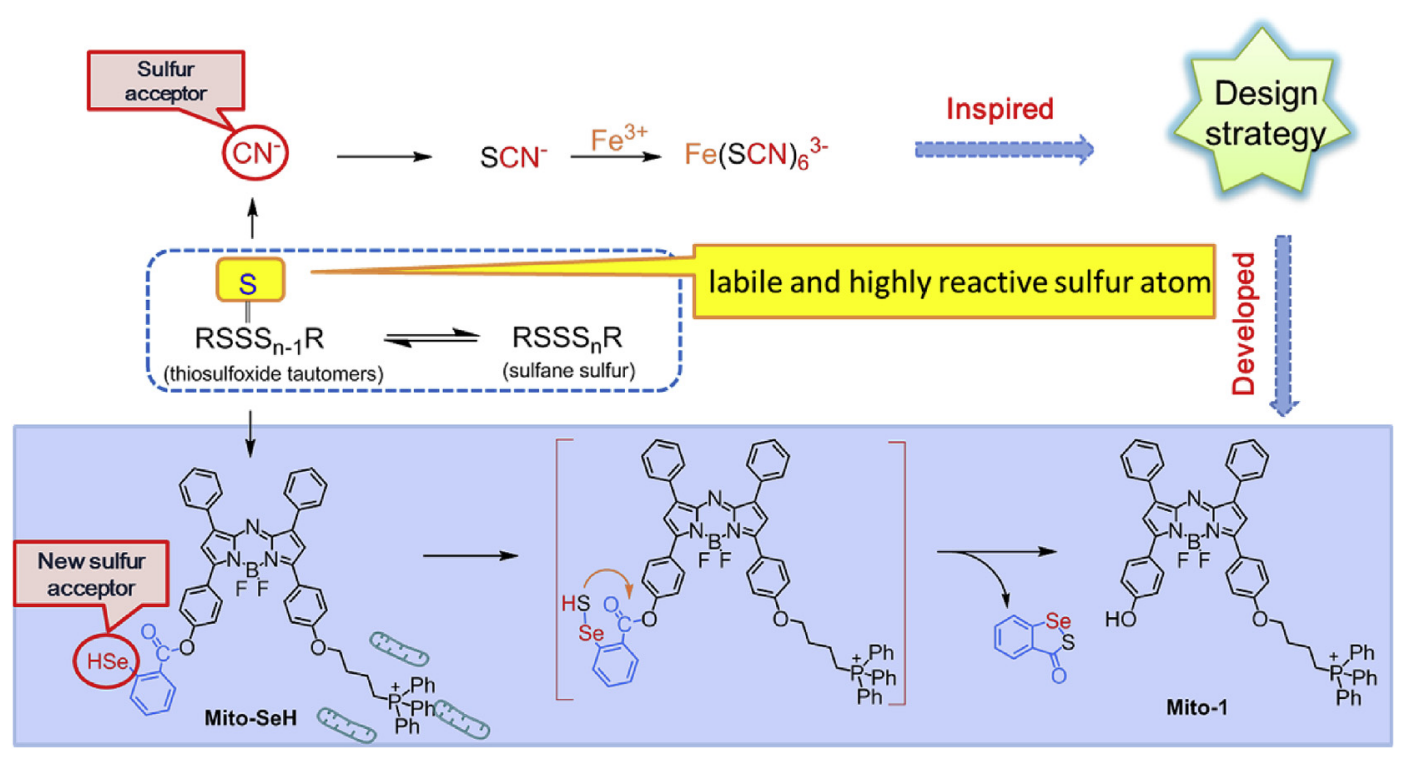

Scheme 1. New design strategy for sulfane sulfur detection. 


\subsubsection{Synthesis of 2,2'-diselanediyldibenzoic acid}

Then 2-amino benzoic acid $(13.7 \mathrm{~g}, 0.1 \mathrm{~mol})$ and $\mathrm{HCl}(1 \mathrm{M}$, $20 \mathrm{~mL}$ ) were added to the mixture and cooled to $0^{\circ} \mathrm{C}$. An aqueous solution of sodium nitrite $(6.9 \mathrm{~g}, 0.11 \mathrm{~mol})$ was added to the mixture and stirred for $1 \mathrm{~h}$. Next an aqueous solution of disodium diselenide $(10.1 \mathrm{~g}, 0.05 \mathrm{~mol})$ was added to the mixture and stirred at $65^{\circ} \mathrm{C}$ for $3 \mathrm{~h}$. Followed at room temperature for another $2 \mathrm{~h}$. Precipitation occurred and the final product was obtained by filtration. The pure product was obtained by recrystallized from acetic acid as a light yellow solid. Yield $68 \% .{ }^{1} \mathrm{H}$ NMR $\left(500 \mathrm{MHz}, \mathrm{CDCl}_{3}-\mathrm{D}_{1}\right) \delta$ (ppm): $12.70(\mathrm{~s}, 1 \mathrm{H}), 8.15(\mathrm{~s}, 1 \mathrm{H}), 7.98-7.97(\mathrm{q}, 2 \mathrm{H}), 7.66-7.64(\mathrm{~d}, 2 \mathrm{H})$, 7.40-7.37 (t, 2H), 7.30-7.27 (t, 2H). LC-MS (ESI ${ }^{-}$): $m / z \mathrm{C}_{14} \mathrm{H}_{10} \mathrm{O}_{4} \mathrm{Se}_{2}$ calcd. 401.8910, found [M-H] $]^{-} 400.8837$.

\subsubsection{Synthesis of Mito-SeH}

Mito-1 was synthesized in our laboratory, the synthesis steps was presented in Supplementary Information [3]. To a mixture of Mito-1 (92.6 mg, $0.1 \mathrm{mmol}), \quad 2,2^{\prime}$-diselanediyldibenzoic acid (40.0 mg, $0.1 \mathrm{mmol}$ ), 1-(3-dimethylaminopropyl)-3-ethylcar bodiimide hydrochloride (EDC, $19.2 \mathrm{mg}, \quad 0.1 \mathrm{mmol}$ ) and 4dimethylaminopyridine (DMAP, $12.2 \mathrm{mg}, 0.01 \mathrm{mmol}$ ) was added $\mathrm{CH}_{2} \mathrm{Cl}_{2}(50 \mathrm{~mL})$ at room temperature. The mixture was stirred for $12 \mathrm{~h}$. Then the mixture was neutralized with dilute $\mathrm{HBr}$, and partitioned between $\mathrm{CH}_{2} \mathrm{Cl}_{2}(50 \mathrm{~mL})$ and $\mathrm{H}_{2} \mathrm{O}(50 \mathrm{~mL})$. Then solvent was evaporated under reduced pressure, the resulted residue was dissolve in ethanol ( $50 \mathrm{~mL}$ ), dithiothreitol (DTT, $15.4 \mathrm{mg}, 0.1 \mathrm{mmol}$ ) was added to the mixture at room temperature. The mixture was stirred for $5 \mathrm{~h}$. Then solvent was evaporated under reduced pressure and the resulted residue subjected to column chromatography for purification $\left(\mathrm{CH}_{2} \mathrm{Cl}_{2}\right)$. Probe Mito-SeH was obtained as a green solid. Yield 30\%. ${ }^{1} \mathrm{H}$ NMR (500 MHz, $\left.\mathrm{CDCl}_{3}-\mathrm{D}_{1}\right) \delta(\mathrm{ppm}):{ }^{1} \mathrm{H}$ NMR (500 MHz, DMSO-D $) \delta(\mathrm{ppm}): 8.27-8.55(\mathrm{~d}, 2 \mathrm{H}), 8.22-8.16(\mathrm{~m}$, $7 \mathrm{H}), 7.91-7.72(\mathrm{q}, 19 \mathrm{H}), 7.58-7.46(\mathrm{~m}, 9 \mathrm{H}), 7.13-7.12(\mathrm{~d}, 2 \mathrm{H})$, $4.23-4.21(\mathrm{t}, 2 \mathrm{H}), 3.75-3.69(\mathrm{~m}, 2 \mathrm{H}), 2.00-1.95(\mathrm{~m}, 3 \mathrm{H}), 1.78-1.71$ $(\mathrm{m}, 2 \mathrm{H}) .{ }^{13} \mathrm{C}$ NMR (125 MHz, DMSO-D $) \delta(\mathrm{ppm}): 164.09,162.45$, 160.86, 160.07, 155.20, 152.63, 146.29, 144.42, 144.11, 141.65, 139.66, $135.40,135.38,134.11,134.03,132.22,131.23,130.77,130.67,129.80$, $129.74,129.51,129.24,129.21,126.08,123.06,122.68,121.64,119.64$, $119.23,118.60,115.61,66.98,29.44,20.32,19.92$. LC-MS (ESI+): $\mathrm{m} / z$ $\mathrm{C}_{61} \mathrm{H}_{48} \mathrm{BF}_{2} \mathrm{~N}_{3} \mathrm{O}_{3} \mathrm{PSe}^{+}$calcd. 1030.2654, found $\left[\mathrm{M}^{+}\right]$1030.2578.

\subsection{Western blot}

Cells were lysed using RIPA buffer supplemented with PMSF (Solarbio, China) and PHosSTOP (Roche, Germany) inhibitors for $30 \mathrm{~min}$. Then the cells were scraped, sonicated, and centrifuged $\left(20,000 \times \mathrm{g}\right.$ at $\left.4{ }^{\circ} \mathrm{C}\right)$ for $15 \mathrm{~min}$. Protein concentrations in the supernatant were determined using the BCA Protein Assay kit (Biogot, China) and equal amounts of protein were electrophoresed on 6-12\% SDS-polyacrylamide gels (Bio-Rad, USA) and transferred to PVDF membranes (Millipore, Germany). Then the membranes were blocked with $7 \%$ milk and blotted with primary antibodies overnight at $4{ }^{\circ} \mathrm{C}$. A horseradish peroxidase (HRP)-conjugated secondary antibody was used to quantify protein and an enhance chemiluminescence $(\mathrm{ECL})$ detection system was used to detect the signals.

\subsection{Preparation of the ex vivo mouse tissue sample}

BALB/c mice weighing 25-30g were provided by Binzhou Medical University. Mice were group-housed on a 12:12 light-dark cycle at $22{ }^{\circ} \mathrm{C}$ with free access to food and water for a week before operation. The protocol followed the code of practice for the care and use of animals of Binzhou Medical University. For hypoxic mouse model, mice were placed in normobaric hypoxic (FIO2 11\%) chambers for 1-5 days. Others were left in atmosphere as the normoxia-exposed animal controls. Mice were then sacrificed and the heart, lung, liver, spleen, kidney, and brain dissected. These organs were washed with PBS for several times. Representative tissue samples (tissue $1 \mathrm{~g}$ in $10 \mathrm{~mL}$ saline) were diluted 10 -fold with an ice-cold saline solution $(0.86 \%)$ and homogenized using an ultrasonic sonifier. Tissue homogenates were centrifuged $(3000 \mathrm{r} /$ min for $10 \mathrm{~min}$ at $4{ }^{\circ} \mathrm{C}$ ), and then the $1 \mathrm{~mL}$ supernatant was diluted to $10 \mathrm{~mL}$ with $10 \mathrm{mM}$ HEPES buffer for fluorescent detection. In addition, the organs were frozen for sectioning. Tissue slice samples were made by section machine (Cryostat machine, Leica, $\mathrm{CM} 3050 \mathrm{~S})$ at $50 \mu \mathrm{m}$ thicknesses.

\subsection{Preparation of mice model of acute ischemia}

The portal vein and renal vein were ligated to induce liver and kidney acute ischemia. The lines pass through the vessel without ligation in mice were set as control. All experimental procedures were conducted in conformity with institutional guidelines for the care and use of laboratory animals, and protocols were approved by the Institutional Animal Care and Use Committee in Binzhou Medical University, Yantai, China. Approval Number: No. BZ2014102R.

\section{Results and discussion}

\subsection{Design strategy of Mito-SeH for sulfane sulfur}

Sulfane sulfur which generally tautomerizes to the thiosulfoxide form has been identified to exert extraordinary regulatory functions in biological systems. The thiosulfoxide bond is weak and the sulfur is easily ejected and transferred to sulfur-acceptor, such as nucleophilic $\mathrm{CN}^{-}$. This property has been applied to the detection of sulfane sulfur via ferric thiocyanate (Scheme 1). Taking advantage of the reactive sulfur atom in thiosulfoxide, the mercapto group $(-\mathrm{SH})$ in thiophenol (pKa 6.5) has been employed as a sulfuracceptor for the fluorescent detection of sulfane sulfur [30]. Now that the reactive sulfur atom in thiosulfoxide can be easily removed by nucleophilic - $\mathrm{SH}$, our solving strategy for sulfane sulfur detection is inspired by another stronger sulfur-acceptor of the selenol group $(-\mathrm{SeH})$ in phenylselenol (pKa 5.9). Selenium and sulfur belong to the same main-group element. The chemical properties of selenium are similar to sulfur. However, $-\mathrm{SeH}$ has been supposed to hold more electrophilicity than $-\mathrm{SH}$. We first synthesize two analogues to enable the advantage of $-\mathrm{SeH}$. As shown in Fig. S1, the selenium-containing analogue provides a faster reaction kinetics than the thiophenol analogue. Benefiting from this design strategy, phenylselenol was introduced into an NIR azo-BODIPY fluorophore as the fluorescent modulator (Scheme 1). Once trapped the reactive sulfur atom in thiosulfoxide, there would form a -Se-SH adducts, then undergone a spontaneous and fast intramolecular cyclization to release the extricated fluorophore (Fig. S20). The lipophilic triphenylphosphonium cation was particularly integrated into azoBODIPY platform for enabling the mitochondrial sublocation of the probe Mito-SeH in cells. The synthetic methodology for Mito$\mathrm{SeH}$ is outlined in Scheme 2.

\subsection{Spectral properties and selectivity}

With the probe Mito-SeH in hand, we first tested its spectroscopic properties under simulated physiological conditions (10 mM HEPES buffer, pH 7.4, 0.5\% DMSO, 0.4\% Tween 80 ). $\mathrm{Na}_{2} \mathrm{~S}_{4}$ was selected as a model of sulfane sulfur, because $S_{4}^{2-}$ could tautomerize to various forms of sulfane sulfur in solution [2,31,42-46]. UV-vis 


$$
\text { Se }+\mathrm{NaBH}_{4} \stackrel{\mathrm{H}_{2} \mathrm{O}, \mathrm{NaOH}, \mathrm{N}_{2} \text {, r.t., } 0.5 \mathrm{~h}}{\longrightarrow} \mathrm{Na}_{2} \mathrm{Se}_{2}
$$

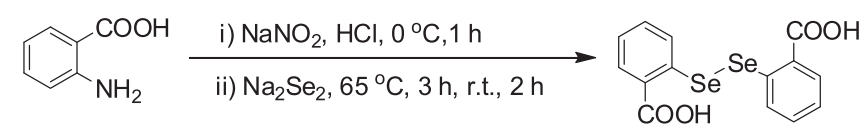

2,2'-diselanediyldibenzoic acid
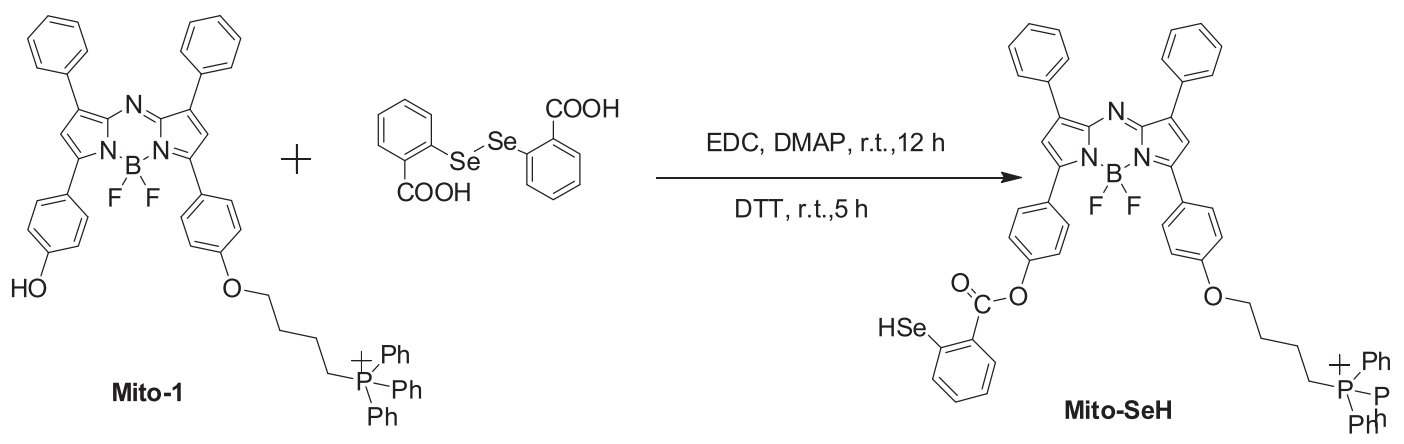

Scheme 2. Synthesis route for Mito-SeH.

spectra of Mito-SeH exhibited one absorption band centered at $680 \mathrm{~nm}$. After treated Mito-SeH with $\mathrm{Na}_{2} \mathrm{~S}_{4}$, a new absorption peak appeared at $688 \mathrm{~nm}$, which was the typical absorption of the fluorophore azo-BODIPY (Fig. 1a). Mito-SeH showed faint fluorescence emission at $720 \mathrm{~nm}$ due to the locked of the two hydroxyl groups of the fluorophore azo-BODIPY. The changes of the fluorescence spectra were further investigated. Upon the gradual addition of $\mathrm{Na}_{2} \mathrm{~S}_{4}$, Mito-SeH provided increasing fluorescent intensities with a
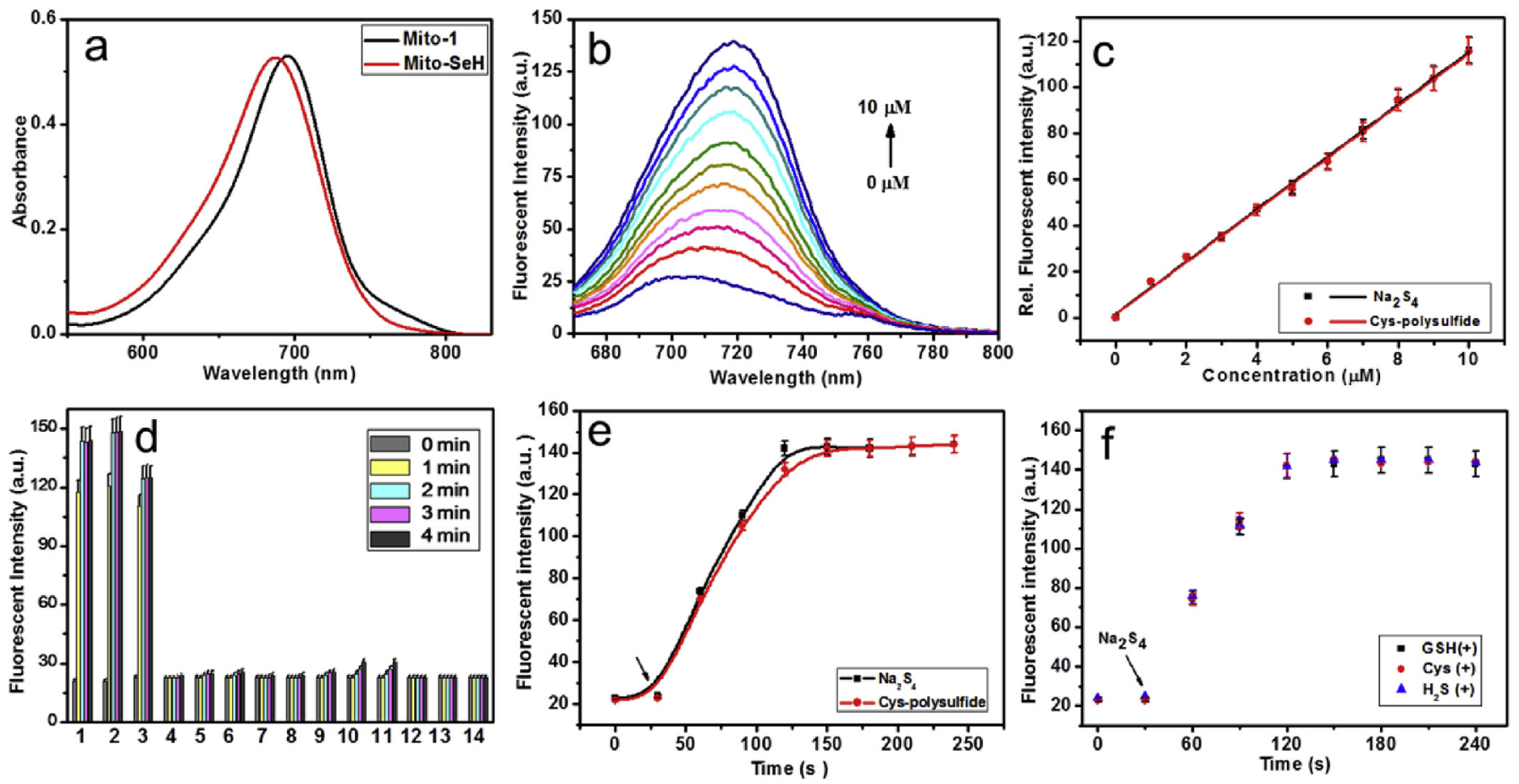

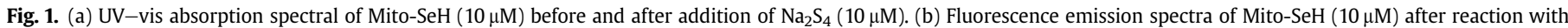

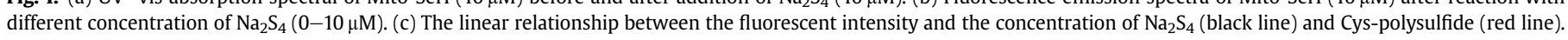

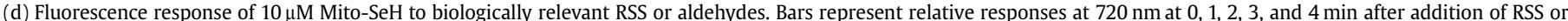

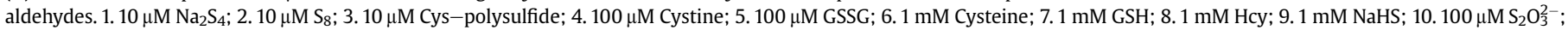

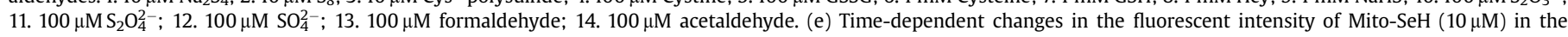

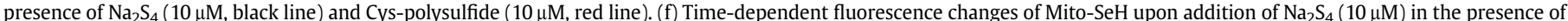

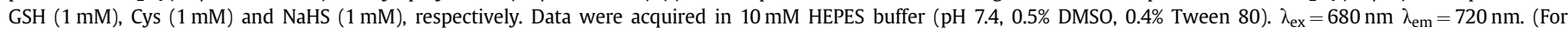
interpretation of the references to color in this figure legend, the reader is referred to the Web version of this article.) 
center at $720 \mathrm{~nm}$ which located in the NIR region (Fig. 1b). And the fluorescent intensities showed a linearly relationship with the concentration of $\mathrm{Na}_{2} \mathrm{~S}_{4}$ from $0 \mu \mathrm{M}$ up to $10 \mu \mathrm{M}$ (Fig. 1c). The regression equation was $\mathrm{F}_{\lambda \mathrm{ex} / \mathrm{em}(680 / 720}$ $\mathrm{nm})=11.36 \times\left[\mathrm{Na}_{2} \mathrm{~S}_{4}\right]+1.779$ with $\mathrm{r}=0.9987$. The limit of detection was determined to be $3.1 \mathrm{nM}(3 \sigma / \kappa)$ under the testing experimental conditions, indicating a high sensitivity for the detection of sulfane sulfur. We also employed Cys-polysulfide as another testing model of sulfane sulfur. As shown in Fig. 1c and e, the test results were almost the same with $\mathrm{Na}_{2} \mathrm{~S}_{4}$. The results demonstrated that probe Mito-SeH could be used to detect sulfane sulfur qualitatively and quantitatively.

Besides sensitivity, selectivity is another important parameter for evaluating the performance of a new fluorescent probe. We now examined the selectivity of Mito-SeH for sulfane sulfur over other reactive sulfur species. As shown in Fig. 1d, Mito-SeH was found to be high selective for sulfane sulfur over other abundant biologically relevant thiols including glutathione (GSH), cysteine (Cys), homocysteine (Hcy) and NaHS. Other relevant RSS, including $\mathrm{S}_{2} \mathrm{O}_{3}^{2-}$, $\mathrm{S}_{2} \mathrm{O}_{4}^{2-}$ and $\mathrm{SO}_{4}^{2-}$ showed almost no fluorescence responses even extend the time to $4 \mathrm{~min}$. The reaction of probe Mito-SeH $(10 \mu \mathrm{M})$ with sulfane sulfur $(10 \mu \mathrm{M})$ at $37^{\circ} \mathrm{C}$ in HEPES buffer $(10 \mathrm{mM}$, pH 7.4, $0.5 \%$ DMSO, $0.4 \%$ Tween 80 ) yielded a time-dependent fluorescence increase. The saturation of fluorescent intensity was reached after incubation of $\mathrm{Na}_{2} \mathrm{~S}_{4}$ for $100 \mathrm{~s}$ (Fig. 1e). The mixture of $\mathrm{H}_{2} \mathrm{~S}$, GSH or Cys in physiological concentration with Mito-SeH could not interfere the detection of sulfane sulfur (Fig. 1f). The result further supported that our probe possessed high selectivity for sulfane sulfur. In addition, the fluorescence responses of Mito-SeH to anions, cations, reactive oxygen and reactive nitrogen species could be neglected (Fig. S2). The effects of $\mathrm{pH}$ on the fluorescent intensity of Mito-SeH were also investigated. The results indicated that the fluorescence responses were stable under simulated physiological conditions (Fig. S3). These features made our probe very attractive for measuring the distribution and contribution of sulfane sulfur in biological systems.

\subsection{Imaging sulfane sulfur and mitochondrial localization}

Since Mito-SeH showed strong ability in detecting sulfane sulfur under simulated physiological conditions, we next assessed whether Mito-SeH was sensitive enough to detect physiologically relevant levels of sulfane sulfur in living cells. Before that, it was necessary to check its biocompatibility. Mito-SeH showed low cytotoxicity toward the vascular smooth-muscle cells (SMCs) as determined via MTT assays (Fig. S4). Photo-bleaching test was performed to evaluate the photostability of Mito-SeH in cells. Fluorescent intensity was stable during the exposure of Mito-SeH to the laser for $500 \mathrm{~s}$. The results showed that Mito-SeH was stable and could be applied for long-time cells imaging (Figs. S5 and S6).

We applied Mito-SeH to living cells for estimating the changes of sulfane sulfur in cells. SMCs in Fig. 2 were divided into seven groups. All the cells were cultured with Mito-SeH for $15 \mathrm{~min}$ at $37^{\circ} \mathrm{C}$. Before image acquisition, the cells were washed with DMEM for 3 times. The intracellular fluorescence in Fig. 2a was very weak. If the cells were pretreated with $\mathrm{N}$-ethylmaleimide (NEM) to deplete all the endogenous RSS, there was almost no fluorescence in Fig. 2b. However, the cells were treated with $\mathrm{Na}_{2} \mathrm{~S}_{4}(1 \mu \mathrm{M})$ for $15 \mathrm{~min}$. The strong fluorescence in these cells was observed (Fig. 2c). The compounds thiophosphate and 3H-1, 2-dithiole-3thione are considered to be the sources of sulfane sulfur in cells. They have been proposed as anticarcinogenic drugs for cancer therapy [2]. After treated with the two compounds, the cells in Fig. $2 \mathrm{~d}$ and e emitted strong fluorescent signals. Taken together, our probe is suitable to directly detect exogenous sulfane sulfur added in living cells. The role of the enzyme CSE in sulfane sulfur generation was evaluated utilizing Mito-SeH. SMCs were stimulated with
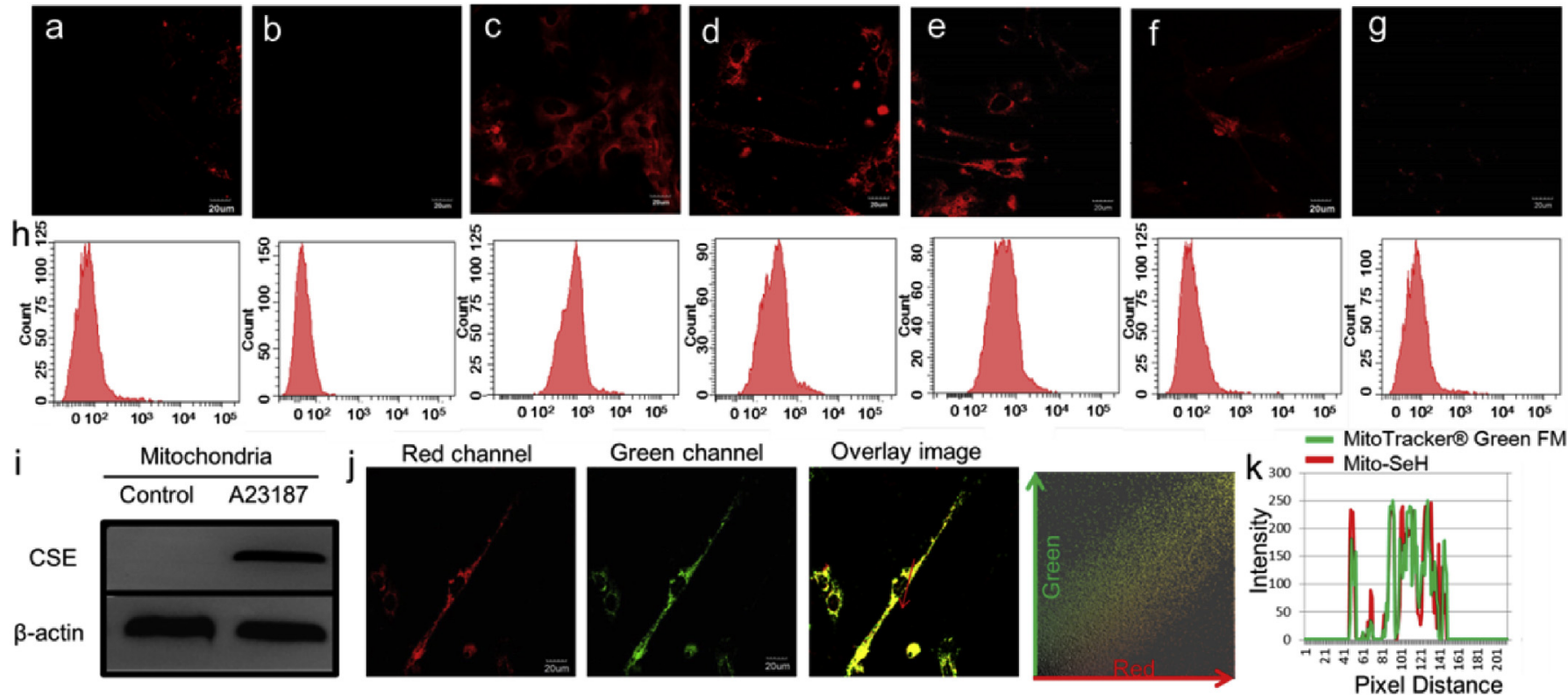

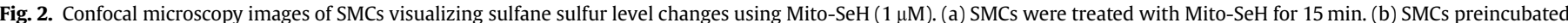

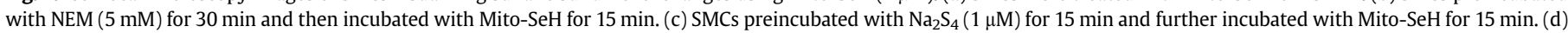

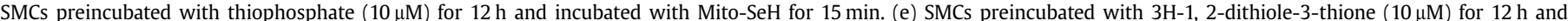

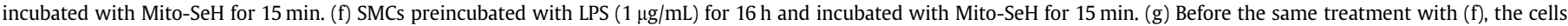

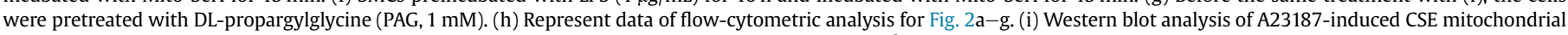

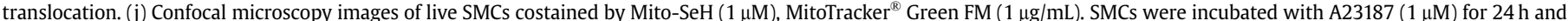

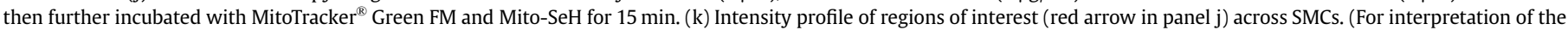
references to color in this figure legend, the reader is referred to the Web version of this article.) 
lipopolysaccharide (LPS) for $16 \mathrm{~h}$ to induce CSE expression [4,5]. As expected, an obvious fluorescent intensity was obtained in Fig. 2f. If pretreated with a CSE inhibitor, DL-propargylglycine $(1 \mathrm{mM})$, the cells showed weak fluorescent signal (Fig. 2 g). In addition, flow cytometry studies were in Fig. $2 \mathrm{~h}$ carried out to further confirm the results of Fig. $2 \mathrm{a}-\mathrm{g}$. The results demonstrated that our probe had potential to detect endogenous sulfane sulfur in cells.

Mitochondria are involved in many crucial cell events. Meanwhile, mitochondria are the main source of ROS within most mammalian cells. The overproduction of ROS contributes to mitochondrial damage in a range of pathologies [47]. The mitochondria fraction contains approximately $60 \%$ of bound sulfane sulfur in cells [10]. Thus, sulfane sulfur has been considered to play protective effects against oxidative stress and maintain cellular redox homeostasis in the antioxidant defense systems. In this work, we anticipated that Mito-SeH should provide a significant mitochondria-targeting ability because of the lipophilic triphenylphosphonium cation moiety. The enzyme CSE in SMCs is mainly localized in cytosol, few in mitochondria. However, CSE can be translocated from cytosol into mitochondria by stimulating with calcium ionophore A23187 (Fig. 2i) [48]. The cells in Fig. $2 \mathrm{j}$ were incubated with $1 \mu \mathrm{M} \mathrm{A23187}$ for $24 \mathrm{~h}$ to accumulate CSE into mitochondria. After loaded Mito-SeH for $15 \mathrm{~min}$, strong fluorescent intensity was observed. Compared with Fig. 2a, the stronger fluorescent intensity illustrated a higher level of sulfane sulfur in mitochondria, which was attributed to the increased concentration of CSE. The data presented here also revealed that A23187stimulated sulfane sulfur production in mitochondria was closely related to the specific catalytic activity of CSE that translocated from cytosol to mitochondria. The cells were costained with $1 \mu \mathrm{g} /$ $\mathrm{mL}$ MitoTracker ${ }^{\circledR}$ Green FM (a mitochondria marker) to assess whether the fluorescence is derived from mitochondria. As shown in Fig. $2 \mathrm{j}$, the images of the two dyes merged well indicating the specifically mitochondrial localization of our probe Mito-SeH in cells. The Pearson's colocalization coefficient was $\mathrm{Rr}=0.97$, and the Manders' coefficients were $\mathrm{m}_{1}=0.99, \mathrm{~m}_{2}=0.98$. The color-pair for each pixel showed a high correlated plot. The changes in fluorescent intensity profiles (Fig. $2 \mathrm{j}$, red arrow) were highly synchronous (Fig. 2k). All the result indicated that our probe Mito-SeH could locate in mitochondria and respond to mitochondrial sulfane sulfur.

\subsection{Sulfane sulfur changes under hypoxia stress}

Appropriate oxygen supply maintains cellular normal activities, while hypoxia causes a series of physiological dysfunctions. Hypoxia stress can inhibit the activities of antioxidant enzymes, which accompanies the apparent increase of oxygen radicals and lipid peroxides. However, sulfane sulfur exhibits antioxidant and cytoprotective effects towards cells. To maintain the balance of intracellular redox milieu, the cells may change the concentration of sulfane sulfur to adapt hypoxic condition. We performed assays in cells to investigate how sulfane sulfur fluctuated under hypoxia stress. The level changes of sulfane sulfur during $8 \mathrm{~h}$ under $0.1 \% \mathrm{O}_{2}$ condition in SMCs were firstly assessed (Fig. S7). The fluorescent intensities were presented as hump profiles. The peak concentration approximately appeared at $4 \mathrm{~h}$, and then decreased. After $6 \mathrm{~h}$, the fluorescent intensity reached platform. The results indicated there existed fluctuations of sulfane sulfur levels over time under hypoxia stress. We next applied the probe to examine intracellular sulfane sulfur changes under different $\mathrm{O}_{2}$ levels. SMCs in Fig. 3a were cultured under hypoxic conditions with $20 \%, 10 \%, 5 \%, 1 \%$, and $0.1 \% \mathrm{O}_{2}$ for $4 \mathrm{~h}$. Strong fluorescent images were acquired from cells cultured with $1 \%$ and $0.1 \% \mathrm{O}_{2}$, while the signal was quite weaker under $10 \%$ and $20 \% \mathrm{O}_{2}$. The degrees of hypoxia were positively correlated with fluorescent intensities of Mito-SeH, revealing that the stress response of sulfane sulfur was strongly dependent on oxygen levels. Flow cytometry studies were carried out to confirm the results in Fig. 3a. These data indicated that our probe could be used to real-time detect hypoxia-induced sulfane sulfur changes in living cells.

Having assessed the trends of sulfane sulfur under different $\mathrm{O}_{2}$ levels, we conducted an investigation to determine the changes of ROS under the same condition using a ROS fluorescent probe Hydro-IR-783 [49]. As shown in Fig. 3b, the fluorescent intensities were gradually increased along with the decreasing oxygen levels. A further analysis was also conducted using flow cytometry to confirm the above results. Taken together, both sulfane sulfur and ROS were upregulated under hypoxic stress during $4 \mathrm{~h}$. The phenomena were attributed to cellular stress response caused by hypoxia. This redox imbalance would result in cell apoptosis. To access the apoptosis, we performed PE Annexin V/7-AAD assay under different degrees of hypoxia. As demonstrated in Fig. 3c, the cells which were cultured under $20 \%, 10 \%, 5 \%, 1 \%, 0.1 \% \mathrm{O}_{2}$ led to the rates of apoptosis were $10.2 \%, 19.5 \%, 28.4 \%, 32.6 \%$ and $35.5 \%$, respectively. The data displayed that hypoxia accelerated cell apoptosis. Moreover, the loss of mitochondrial membrane potential of the cells under hypoxia stress was increased by using JC- 1 (Fig. S8b). In addition, the cells under hypoxia stress induced caspase-dependent apoptosis (Fig. S8c). Next, the expressions of cleaved caspase 3 , cytochrome $c$, poly (ADP-ribose) polymerase (PARP), hypoxia inducible factor $-1 \alpha$ (HIF-1 $\alpha$ ) were examined by western blot analysis (Fig. S8d). These results demonstrated that hypoxia stress could induce cytochrome $c$-dependent caspase activation, which was initiated from mitochondria.

\subsection{Cytoprotective effects of sulfane sulfur}

We had confirmed that hypoxia stress could stimulate mitochondrial ROS burst and induce cell apoptosis. Sulfane sulfur was able both to scavenge ROS and to maintain cellular redox homeostasis. We next explored whether sulfane sulfur possessed the capability of cytoprotection against oxidative damage under hypoxia stress (Fig. S9). To determine the potential effects of sulfane sulfur on eliminating hypoxia-induced intracellular ROS, we employed the probe Hydro-IR-783 for indicating the levels of ROS. As shown in Fig. 4a, the cells in group a - c were cultured under normoxic condition $\left(20 \% \mathrm{O}_{2}\right)$, while the cells in group $\mathrm{d}-\mathrm{f}$ were cultured under hypoxic condition $\left(0.1 \% \mathrm{O}_{2}\right)$ for $4 \mathrm{~h}$. The cells in each cultured conditions were pretreated with the sulfane sulfur source $3 \mathrm{H}-1,2$-dithiole-3-thione 0,100 , and $300 \mu \mathrm{M}$, respectively. The fluorescent intensities of each groups indicated the levels of intracellular ROS. Clearly, hypoxia stress led to the burst of ROS. The addition of sulfane sulfur could eliminate ROS resulting in attenuated fluorescence (group e and f). Interestingly, the group c offered a slightly bright fluorescence image suggesting the generation of ROS. The reason was ascribed to the severely disturbed intracellular redox homeostasis by excessive exogenous sulfane sulfur. The cells had no choice but produced ROS against the temporary reducing stress. The changes in fluorescent signal were also proved via flow cytometry assay (Fig. 4). The results implied that sulfane sulfur could prevent cell against hypoxia-induced ROS damage. However, the excessive dose of sulfane sulfur would cause ROS increase.

Cell apoptosis was assessed using flow cytometry analysis. Sulfane sulfur obviously decreased the apoptosis rate of cells under hypoxic condition (Fig. 4b). The apoptosis rates were sorted as: Group $\mathrm{f}<$ Group e $<$ Group $\mathrm{d}$. The apoptosis rates in normoxic condition were no obvious differences, despite a high level of ROS was detected. Mitochondrial membrane potential was further assessed using JC-1. The apoptotic trends were well consist with PE Annexin V/7-AAD assay (Fig. 4c). Transmission electron microscopy 


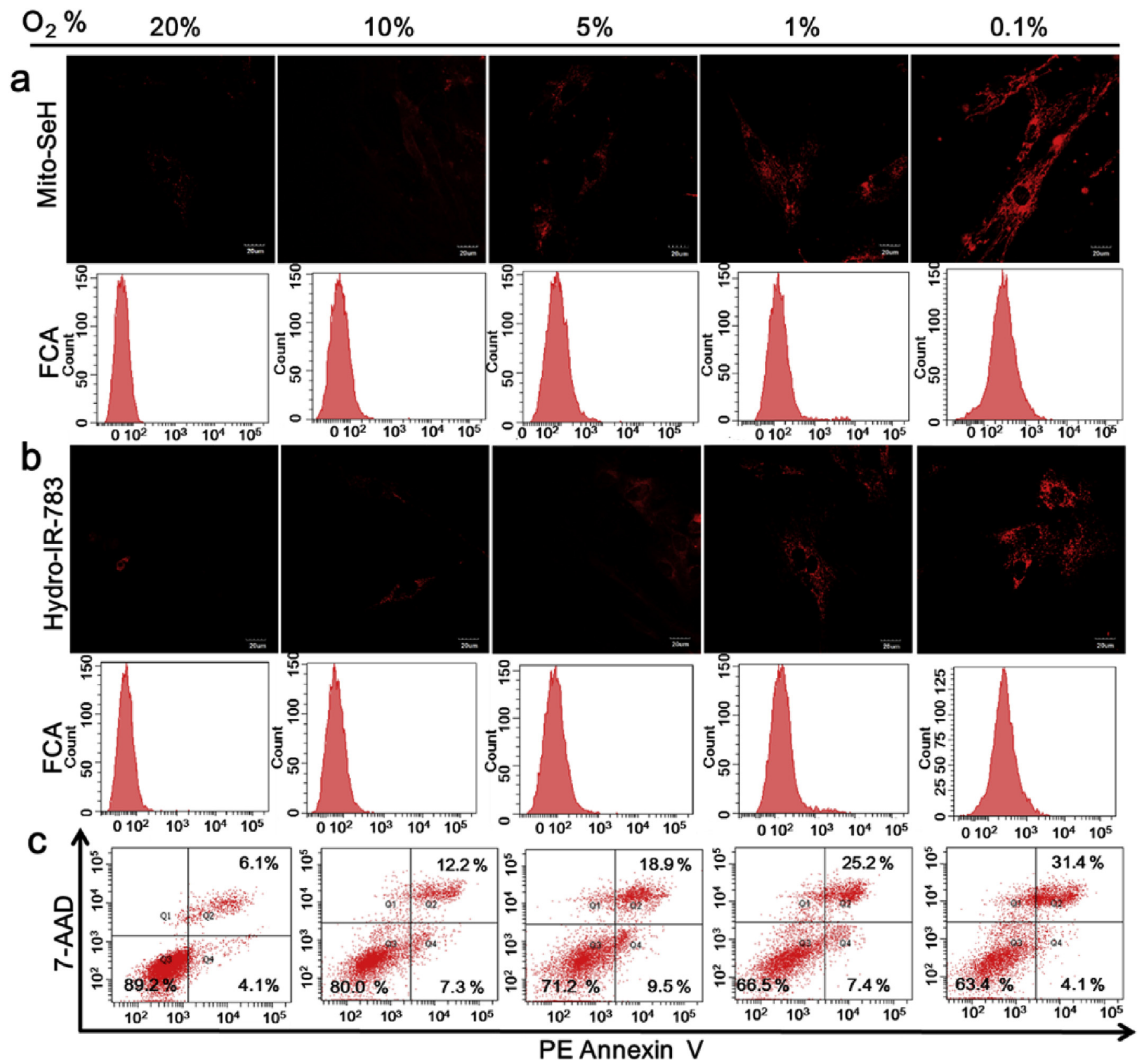

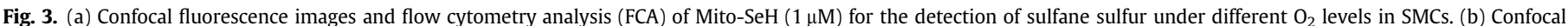

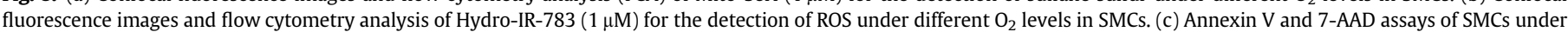

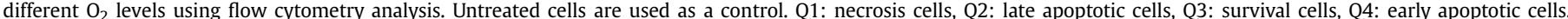

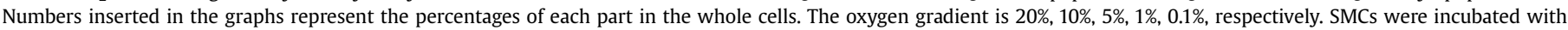

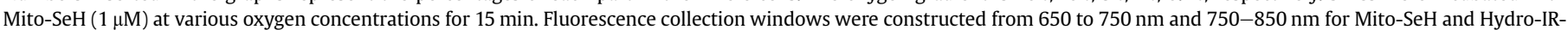
783 , respectively.

(TEM) was next applied for observing cell morphology. Cells cultured under normoxic condition showed no significant difference in apoptosis (Group a - c). However, hypoxia stress gave rise to characteristic apoptotic morphology in cells of Group d, such as increased lipid droplets and endoplasmic reticulum expansion (Fig. 4d). But the cells which were pertreated with sulfane sulfur (Group e and f) had improved these symptoms. The expression of cytochrome c in cytosol, cleaved-caspase 3, cleaved PARP, and HIF$1 \alpha$ also decreased after pretreatment with sulfane sulfur under hypoxia stress (Fig. 4e). All the above results provided a fact that sulfane sulfur played significant antioxidant and cytoprotective roles during hypoxic process. These findings of the present study had demonstrated for the first time that sulfane sulfur protected SMCs against hypoxic damage via inhibiting ROS-activated caspasedependent apoptosis in mitochondria (Fig. 4f).

\subsection{Distribution of sulfane sulfur in hypoxic models}

As the degrees of hypoxia stress influenced the generation of sulfane sulfur in cells, we wanted to map the distribution of sulfane sulfur along with gradual $\mathrm{O}_{2}$ attenuation in hypoxic models. The oxygen gradient was manipulated by mounting a quartz thin cover 


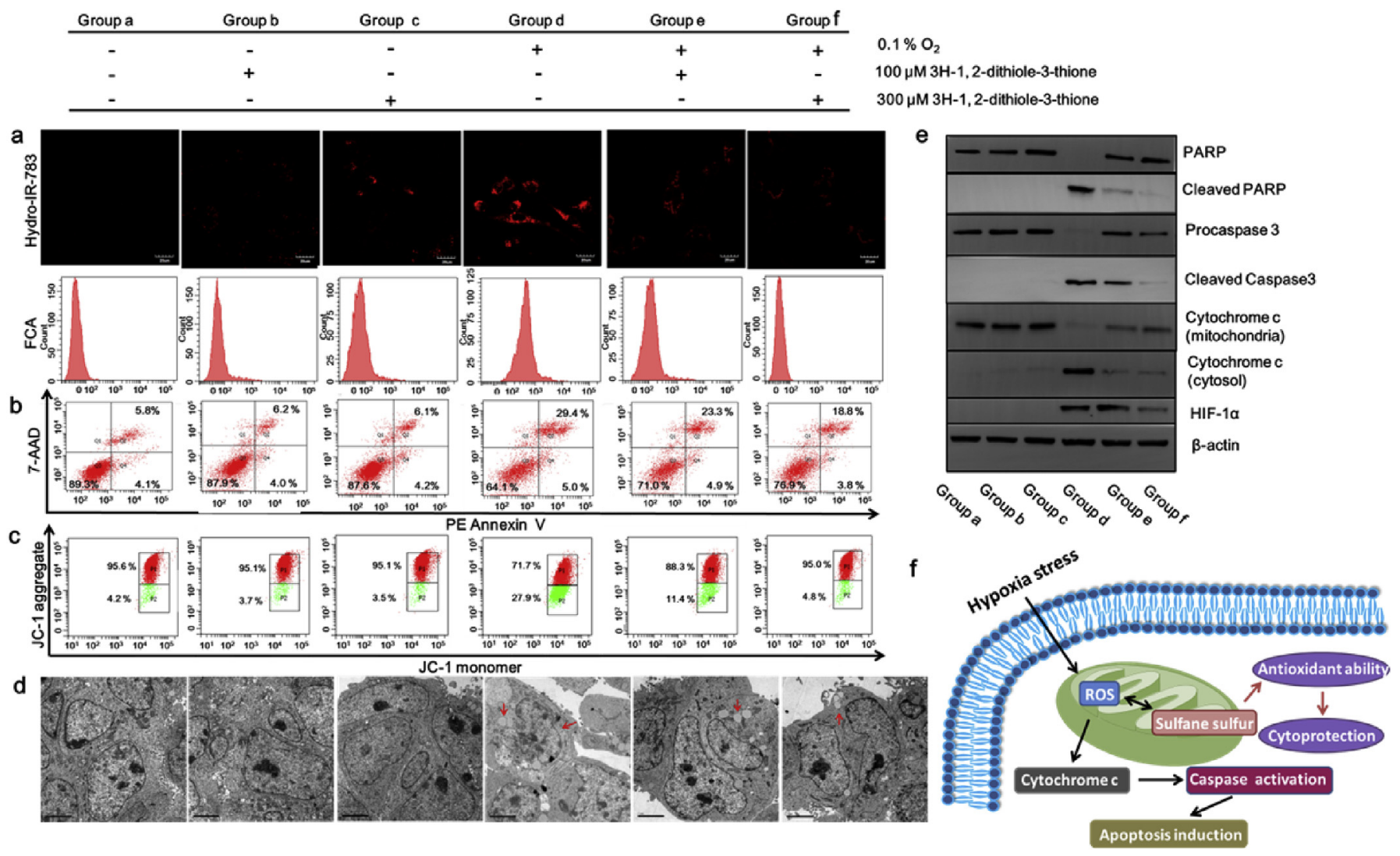

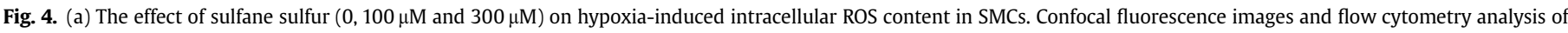

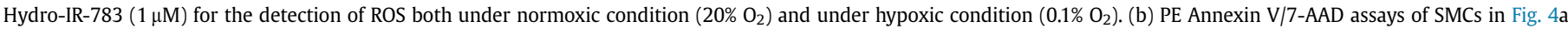

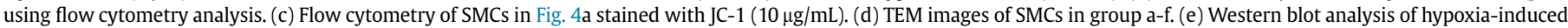

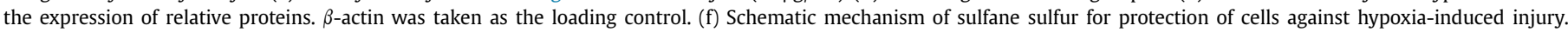
Fluorescence collection windows were constructed from 750 to $850 \mathrm{~nm}$.

glass on top of culture cells (Fig. 5a) [50-52]. Once blocked $\mathrm{O}_{2}$ diffusion from above, the concentration of $\mathrm{O}_{2}$ gradually decreased with the distance from the edge to the center of the slide. SMCs were incubated Mito-SeH and Hoechst 33,342 under normal culture conditions, then covered a slide on top for $4 \mathrm{~h}$ to create oxygen gradient. As expected in Fig. 5a, the cells at the center of the slide provided strong fluorescence, while the cells at the edge emitted faint fluorescence. The signal intensity profile displayed the relationship between $\mathrm{O}_{2}$ level and the concentration of sulfane sulfur along with the distance from the edge to center (Fig. 5b). The results revealed that our probe Mito-SeH allowed to image cellular hypoxia-dependent sulfane sulfur.

Now that we had evaluated the response behavior of Mito-SeH in monolayer cells under hypoxia stress. We next constructed three-dimensional multicellular spheroid (3D-MCs) to further assess the relationship between sulfane sulfur and hypoxia. The interior of 3D-MCs was a hypoxic micromilieu because of the limited oxygen diffusion. As shown in Fig. $5 c$, after incubated with Mito-SeH for $8 \mathrm{~h}, 3 \mathrm{D}-\mathrm{MCs}$ offered a strong fluorescence from the core, which indicated the production of sulfane sulfur in interior. No fluorescence was observed in the periphery of 3D-MCs where exposed to the $\mathrm{O}_{2}$ saturated culture medium. The signal intensity profile in Fig. $5 \mathrm{~d}$ showed the quantitative and spatial distribution of fluorescence emission across the green line in Fig. 5c. Z-stack reconstruction and images for 3D-MCs showed activation of fluorescence signal even at a depth of $130 \mu \mathrm{m}$, which indicated Mito$\mathrm{SeH}$ could penetrate into the core and response to sulfane sulfur. The stereoscopic fluorescent image of hemispherical 3D-MCs was established from multi-perspective view, which further confirmed that the fluorescence signal was from the interior of 3D-MCs (Fig. 5e). All the results demonstrated that the hypoxic state within 3D-MCs could cause an increase in the sulfane sulfur concentrations.

\subsection{Imaging and quantification of sulfane sulfur in vitro assay}

Having demonstrated the capability of Mito-SeH for sulfane sulfur detection in complicated 3D-MCs, we assessed the applications of Mito-SeH to respond to sulfane sulfur in vitro and in vivo. As shown in Fig. S10, the probe Mito-SeH could detect sulfane sulfur in mouse peritoneal cavity. We next inspected the effects of environmental hypoxia stress on organs of mice, especially the mutual relationship between sulfane sulfur and ROS. The female BALB/C mice were divided into six groups and placed in normobaric hypoxic chambers (Fraction of inspiration $\mathrm{O}_{2}$, FIO2 11\%) for $0-5$ days to create hypoxic mouse models before requiring sacrifice (Fig. S19). Then samples of heart, liver, lung, spleen, kidney, and brain were carefully isolated. The fluorescence imaging of hypoxic mouse tissues slices stained with Mito-SeH were acquired. As shown in Fig. 6a, only brain and kidney gave moderate fluorescence emission in control group ( 0 day). Strong fluorescence were observed in liver and spleen tissue of the mice in normobaric hypoxic chambers for the 1 st day, followed by a sharp decrease in fluorescent intensity at the 2nd day. Then gradual increases in fluorescent signals were obtained in all tissues from the 2nd day to the 4th day. At the 5th day, the fluorescent signals approximately returned to the original 

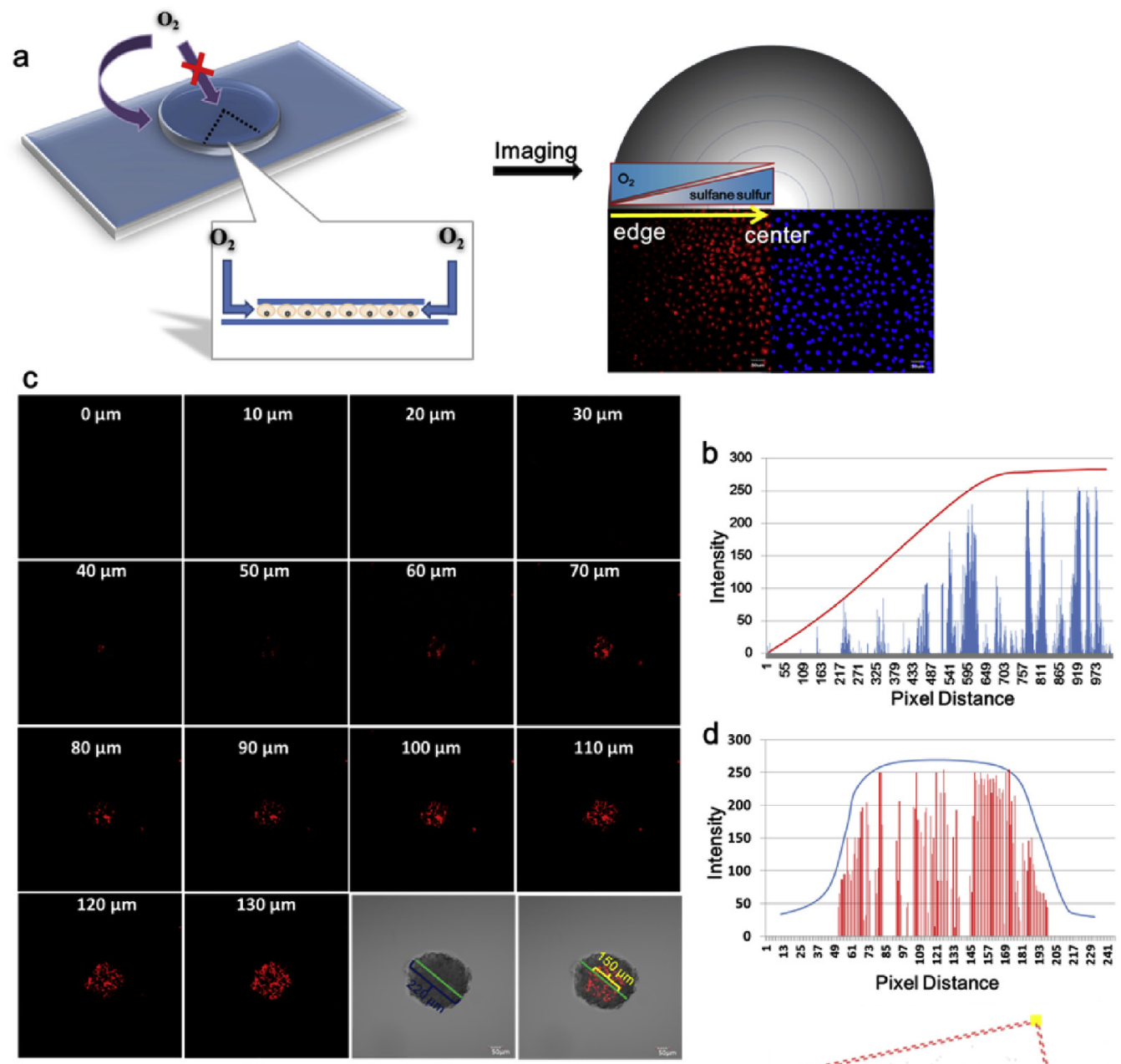

e
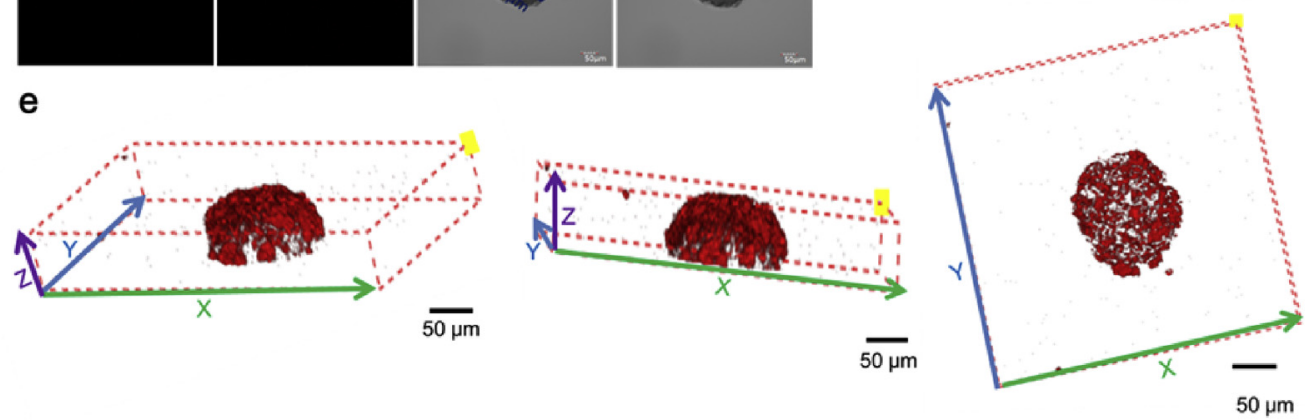

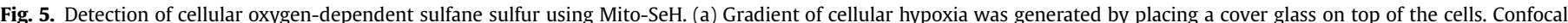

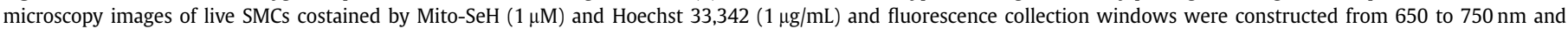

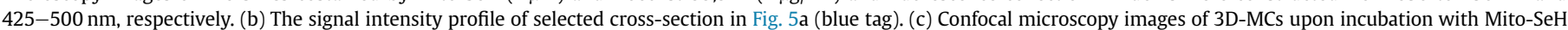

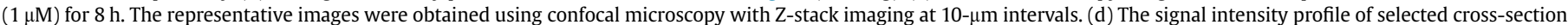

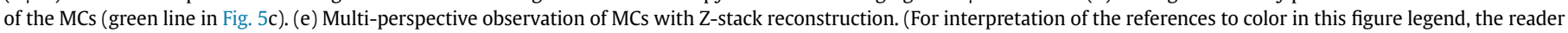
is referred to the Web version of this article.)

level (0 day). To accurately understand the sulfane sulfur changes caused by hypoxia stress, we applied Mito-SeH to determine sulfane sulfur concentrations in tissue homogenates. The data were shown in Fig. 6b. Obviously, hypoxia stress could induce severe fluctuations of sulfane sulfur. However, the changes in each organ are not the same. The hypoxic simulation for one day led to the increase of sulfane sulfur in heart, liver and spleen, while those of kidney, lung, and brain decreased. But the concentration of sulfane sulfur fell sharply to the bottom indicating excessive depletion of sulfane sulfur for stressing two days. Changes in the lung was an exception, it offered a rapid response to hypoxia stress. Starting from the 3rd day, the organs began to supplement sulfane sulfur against hypoxia stress, and reached peak at the 4th day. Sulfane sulfur in each organ at the 5th day was roughly stable to physiological concentrations, which illustrated the self-limiting response to prevent excessive depletion of the pool. Whereas, sulfane sulfur in heart undergone a gradual growth process. The average concentrations of sulfane sulfur in each organ were calculated and shown in Fig. 6c. HPLC method was performed to confirm these results (Fig. S11). These results demonstrated that our probe MitoSeH could exactly trap the level changes of sulfane sulfur. The pool of sulfane sulfur in vivo could be disturbed by hypoxia stress. We 

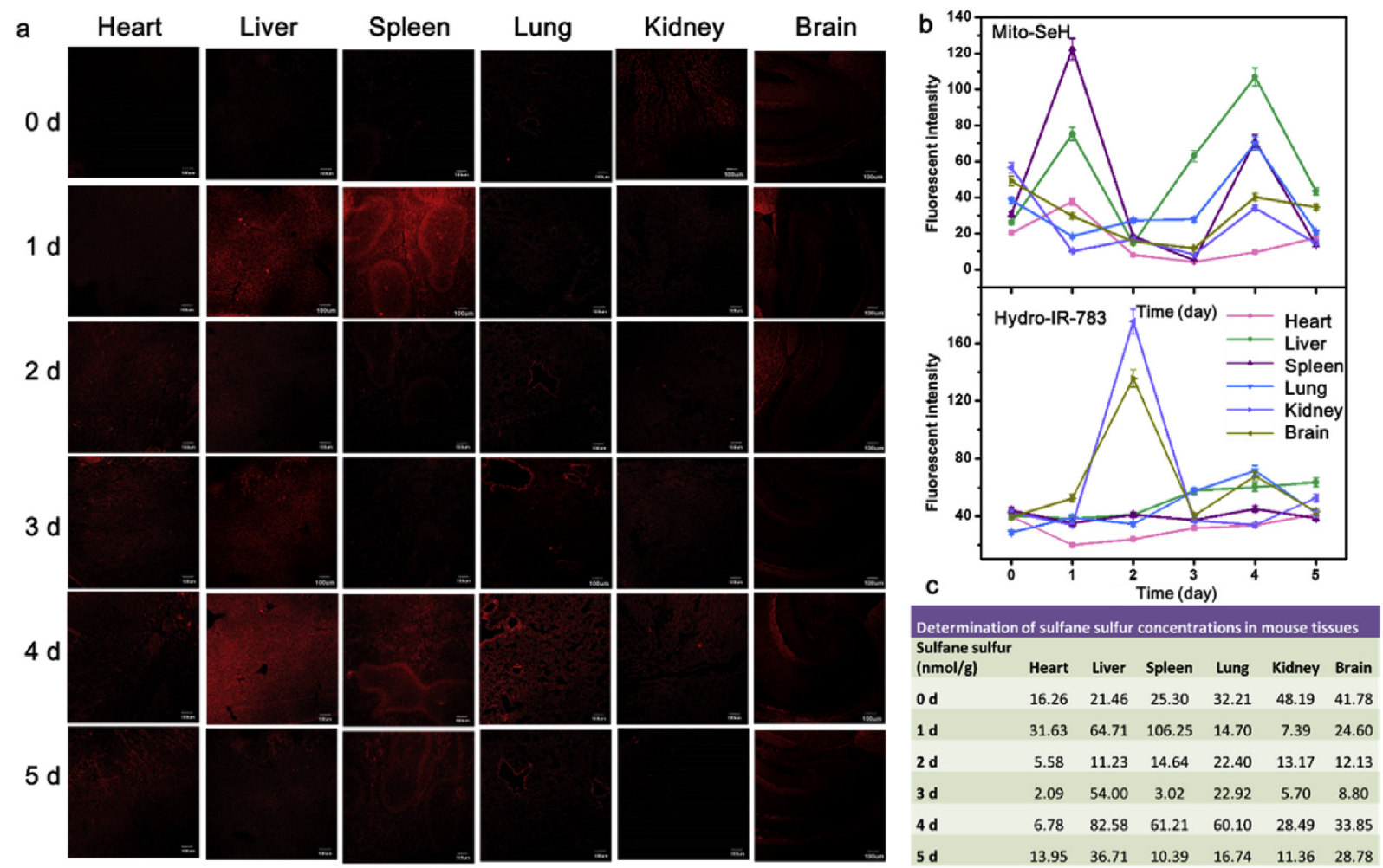

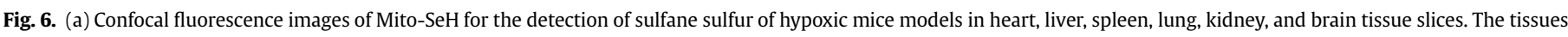

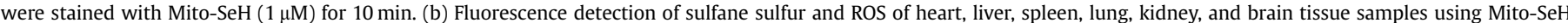

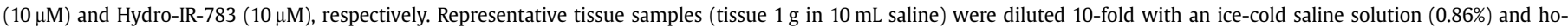

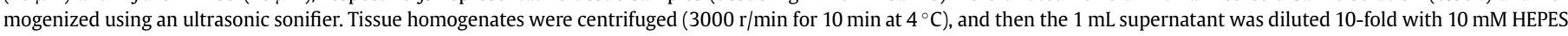

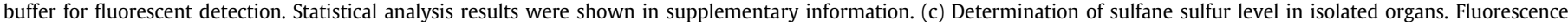
collection windows were constructed from 650 to $750 \mathrm{~nm}$ and $750-850 \mathrm{~nm}$ for Mito-SeH and Hydro-IR-783, respectively. Data are presented as means \pm SD ( $\mathrm{n}=5$ ).

directly observed organism self-regulation in response to hypoxia stress in terms of real-time changes of sulfane sulfur. In view of the relationship between sulfane sulfur and ROS in living cells, we constructed an additional experiment to determine the interaction of this redox couple in different organs of mice under hypoxic condition. The ROS level in isolated organs was determined by a ROS fluorescent probe Hydro-IR-783. The changes of fluorescent intensities in each organ over time were displayed to allow for direct comparisons (Fig. 6b). We found that the level changes between sulfane sulfur and ROS was approximately identified as the negative ratio. Although, kidney and brain showed burst productions of ROS at the 2nd day, suggesting poor tolerance of the two organs to hypoxia stress. Overall, the level of ROS increased slowly, which was attributed to the protective effects of sulfane sulfur on scavenging overproduced intracellular ROS. All these experiments established that our probe was a qualified candidate for the real-time detection of sulfane sulfur in isolated organs of hypoxic mouse modal, which may help us to clarify the relationship between sulfane sulfur and ROS under external stimuli conditions.

\subsection{Imaging sulfane sulfur in acute ischemia model}

Ischemia of organs will block blood supply and lead to deficiency of $\mathrm{O}_{2}$. Then the normal functions of cells and organs must be affected. The pathogenesis of acute ischemia involves in multiple mechanisms including excessive production of ROS. Once acute ischemia occurs, these ROS will irreversibly damage biomacromolecules, which results in cell dysfunction. We next employed Mito-SeH to examine the changes of sulfane sulfur in mice model of acute ischemia. Meanwhile, the variation of ROS was also detected. As displayed in Fig. 7, the mice were intravenously injected with Mito-SeH and Hydro-IR-783 $(10 \mu \mathrm{M}, 50 \mu \mathrm{L}$ in 1:9 DMSO/saline $v / v)$, respectively. The fluorescent images were acquired using in vivo imaging system. The models were divided into two groups: I) acute ischemia group and II) therapy group. After $1 \mathrm{~h}$ injection of probes, the portal vein and renal vein were ligated to induce acute ischemia. The mice without vessel ligation were set as control. In I group, the fluorescence signals of Mito-SeH steeply increased and saturated at the time point of $60 \mathrm{~min}$, followed by decreasing in intensities (Fig. 7a). Whereas the fluorescent signals of Hydro-IR-783 gradually raised, indicating the burst of ROS under hypoxic conditions (Fig. 7b). The result illustrated that sulfane sulfur should be rapidly released to scavenge the overproduction of ROS in acute ischemia. But the generation of endogenous sulfane sulfur was limited under ischemia. In II group, the mice were intravenously injected with $3 \mathrm{H}-1$, 2-dithiole-3-thione $(200 \mu \mathrm{M})$ to supplement sulfane sulfur before ligation. We gathered the strong fluorescent images both in liver and kidney over time due to the adequate pool of sulfane sulfur (Fig. 7c). Therefore, the ROS were controlled at low level (Fig. 7d). The corresponding fluorescence quantitative data were displayed in Fig. $7 \mathrm{e}-\mathrm{h}$. The fluorescence changes in the above four operations were also offered as movies (Video a - d in SI). The rapid response time and the highly sensitivity made our probe a useful chemical tool for real-time monitoring changes of sulfane sulfur in acute ischemic mice model.

Supplementary video related to this article can be found at https://doi.org/10.1016/j.biomaterials.2018.01.011. 


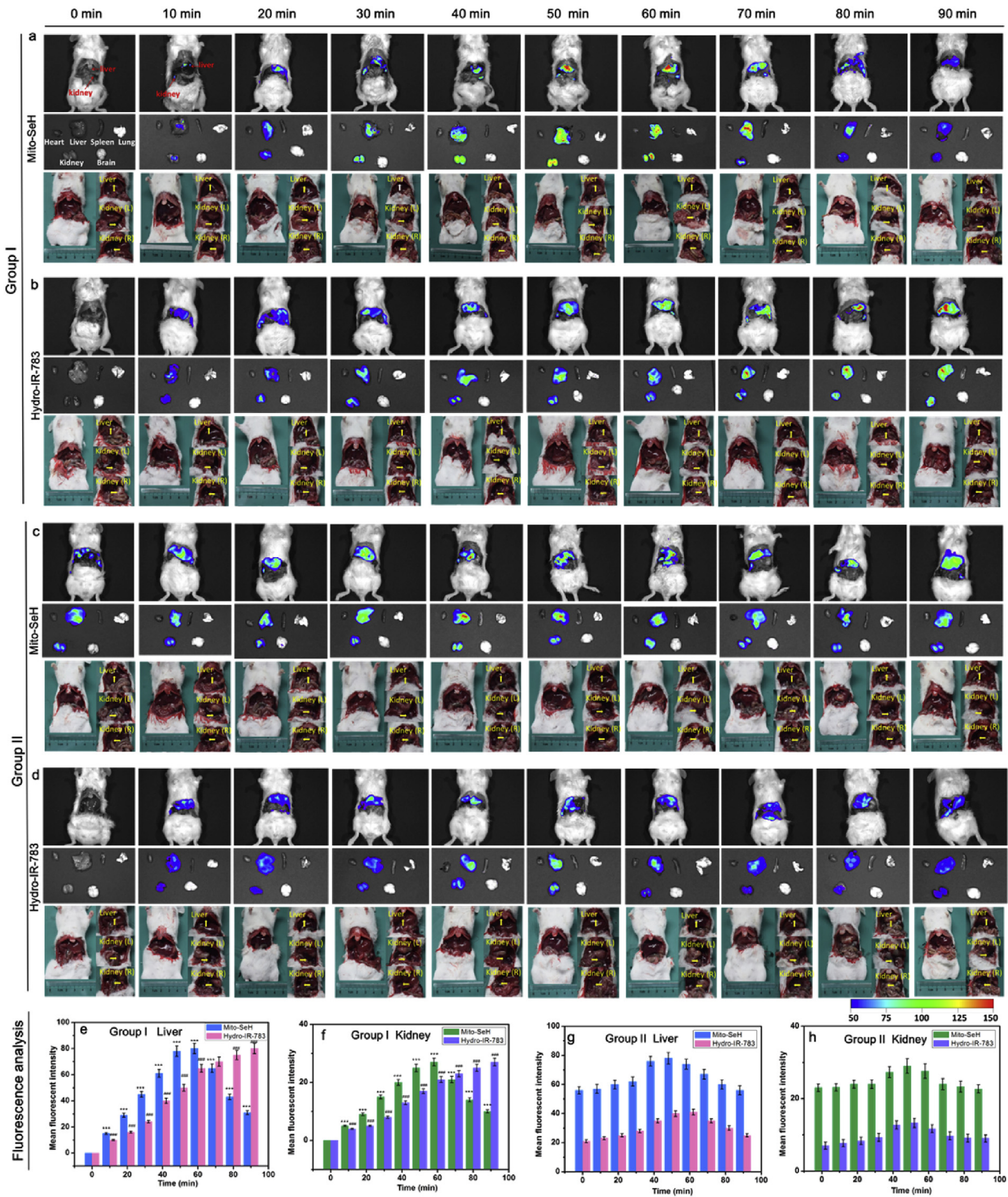

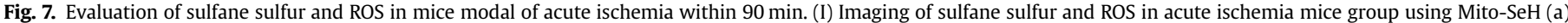

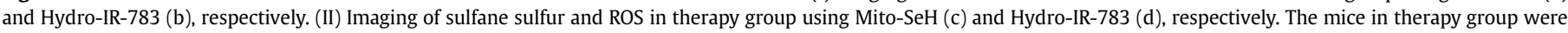

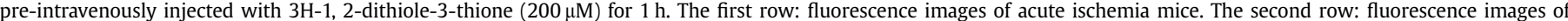

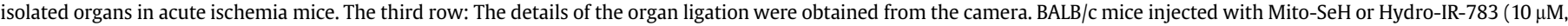

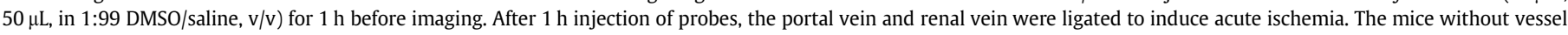

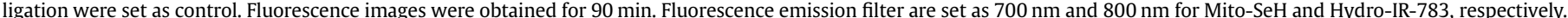

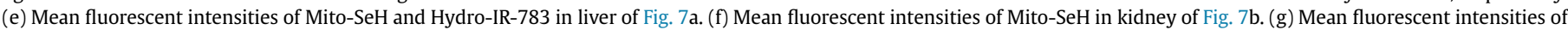

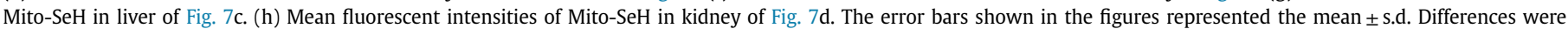

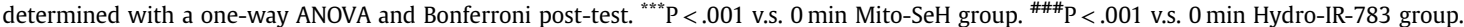




\section{Discussion}

Oxygen is crucial to cell survival and cell activities. Hypoxia is a pathological oxygen-deprived state, which can disrupt the homeostasis of intracellular redox milieu and lead to severe cell oxidative damage. The overproduction of reactive oxygen species (ROS) under hypoxic condition is closely associated with various diseases, including cardiac ischemia [53-55], inflammatory diseases [56], and tumors [57,58]. However, to against hypoxia stress, the cells struggle to maintain the intracellular redox homeostasis through their intrinsic antioxidant regulatory systems. Sulfane sulfur is one of the crucial contributors of antioxidant defense systems in cells. Sulfane sulfur generally presents in forms of persulfides ( $R S_{n} S H, n \geq 3$ ), polysulfides $\left(R-S_{n}-R, n \geq 3\right.$ ), hydrogen polysulfides $\left(\mathrm{H}_{2} \mathrm{~S}_{\mathrm{n}}, \mathrm{n} \geq 2\right)$, and elemental sulfur $\left(\mathrm{S}_{8}\right)$. Antioxidant activities associated with sulfane sulfur are attributed to increase the activities antioxidant enzymes, scavenge radicals and enzymatically decompose oxygen metabolites [18,19,59]. However, sulfane sulfur exhibits strong nucleophilic/antioxidant activities. The derivatives of sulfane sulfur are proposed to be direct antioxidants against reactive oxygen species in cells, which may provide a primary and potent antioxidant defense in cells [4,60].

Sulfane sulfur and its thiosulfoxide tautomers are coexist in biological systems. The thiosulfoxide tautomers can offer a labile and highly reactive sulfur atom which is beneficial for the detection of sulfane sulfur. The previous detection method is based on the reaction with $\mathrm{CN}^{-}$to yield thiocyanate which is further measured as ferric thiocyanate. Although this colorimetric method is convenient, but it requires sample preparation, including cell lysate or tissue destruction. It cannot meet the applications for the real-time and in situ sulfane sulfur detection in biological samples. Moreover, the concentration of sulfane sulfur is in dynamic changes. Fluorescence imaging technology based on fluorescent probe affords high sensitivity, real-time imaging, and high spatiotemporal resolution, which has developed as an indispensable chemical tool for the non-invasive analysis of intracellular reactive species and a variety of biological events. We integrate a stronger sulfur-acceptor selenol group into a NIR azo-BODIPY fluorophore to construct a fluorescent probe Mito-SeH for imaging and evaluation of sulfane sulfur bioeffects under hypoxia stress (Scheme 1). With the help of selenol group ( $-\mathrm{SeH}$ ) as sulfane sulfur response unit, the probe Mito-SeH has been be applied for the selective detection of sulfane sulfur qualitatively and quantitatively (Fig. 1) with a limit of detection is $3.1 \mathrm{nM}$ under the testing experimental conditions.

Mitochondria has been recognized as one of the main organelle destroyed by the excessive reactive oxygen species (ROS). The mitigation of ROS by mitochondria-targeted antioxidants can inhibit cell apoptosis and prevent aging. Sulfane sulfur can be endogenously produced by CSE [3,4], CBS [5], and 3-MST [6]. To verify the mitochondria-targeting ability of the probe Mito-SeH, the vascular smooth-muscle cell line (SMCs) is chosen as testing model. The enzyme CSE of SMCs mainly localizes in cytosol, few in mitochondria. However, CSE can be translocated from cytosol into mitochondria by stimulating with calcium ionophore A23187 (Fig. 2). The experimental results confirm that our probe can be employed for imaging of endogenous sulfane sulfur fluctuations in mitochondria.

As far as we know, this is the first NIR fluorescent probe for evaluating the level changes of sulfane sulfur under hypoxic stress. As shown in Fig. 3 and Fig. S8, hypoxia stress interferes the homeostasis of associated intracellular redox species. The levels of sulfane sulfur and ROS are positively correlated with the degrees of hypoxia $\left(\mathrm{O}_{2} \%: 20 \%, 10 \%, 5 \%, 1 \%, 0.1 \%\right)$ during the former $4 \mathrm{~h}$. However, the last $4 \mathrm{~h}$ hypoxic stress leads to the concentration of sulfane sulfur decreases. We also access the level changes of the enzyme
CSE in cytoplasm and in mitochondria. The results display that hypoxic stress can induce the overexpression of CSE both in cytoplasm and mitochondria (Fig. S8a). Owing to the lower level of CSE in mitochondria, we suppose that sulfane sulfur produced by the cytoplasmic CSE enzyme must be transported into mitochondria via some mechanisms to help mitochondrial antioxidant. Oxidative stress induced by hypoxia stress will promote cell apoptosis, while sulfane sulfur can protect cells by eliminating ROS. As illustrated in Fig. 4, the addition of sulfane sulfur obviously decreases the cell apoptosis rate. The two cell hypoxic models in Fig. 5 also demonstrate that the production of sulfane sulfur is dependent on the degrees of hypoxia.

We next assess the effects of environment hypoxia stress on the relationship between sulfane sulfur and ROS in various organs of mice. For in vivo experiment, the changes of sulfane sulfur and ROS in each organ are evaluated over hypoxic time (Fig. 6). The level changes between sulfane sulfur and ROS in each organ are different. But the tendency is approximately identified as the negative ratio (Fig. 6b). Compared to the cells, the body is a more complex biological system. There exists various feedback mechanisms and regulatory mechanisms, which are distinct from those in cells. It is also possibly that the mice may adapt to the hypoxic condition after several days. In addition, each organ features its distinct functions and self-protection ability, which may results in the different changes between sulfane sulfur and ROS. Ischemia is a pathological condition which is limited blood supply to a certain area of tissue. The mechanisms of ischemia and hypoxia are not exactly the same. During the acute ischemia process, sulfane sulfur must be rapidly released to scavenge the overproduction of ROS (Fig. 7). However, the generation of endogenous sulfane sulfur is limited over ischemic time, which results in a decrease in sulfane sulfur concentration and an increase in ROS concentration. Evidently, the additional sulfane sulfur efficaciously dominate the levels of ROS. It seems that the results of acute ischemia are consistent with those of cell assays. It is worth noting that the in vitro experiment, acute ischemia and the in vivo hypoxia assay all can lead to crossresponse between sulfane sulfur and ROS, but the conditions of the three experiments are different. We also strive to summarize the results, but it is difficult to make a clear comparison for the above experimental results. We will continue to investigate the protection mechanism of sulfane sulfur against ROS in the future work.

\section{Conclusions}

In summary, we have developed a NIR fluorescent probe, Mito$\mathrm{SeH}$, for sulfane sulfur detection in living cells and in vivo under hypoxia stress. This mitochondria-targeting fluorescent probe showed turn-on fluorescence response to sulfane sulfur over a variety of RSS. The probe is sensitive, stable, and biocompatible, allowing fluorescence imaging of intracellular sulfane sulfur. We measured the changes of sulfane sulfur in monolayer cells, in threedimensional multicellular spheroid, as well as in ex vivo-dissected organs of hypoxic mice modal including heart, liver, spleen, lung, kidney and brain. We established acute ischemia models of mice to evaluate the biological effects of sulfane sulfur on eliminating the overproduction of ROS under hypoxia stress. All the results have disclosed that sulfane sulfur can prevent cells from oxidative damage when suffering from hypoxia stress both in living cells and in vivo. The protective mechanism against hypoxia is to inhibit caspase-dependent apoptosis through scavenging ROS pathway in mitochondria. Our study provides a fact that sulfane sulfur may be a novel therapeutic drug for hypoxia-induced injury. The new probe offers us a valuable tool for further explore physiological and pathological bio-roles of sulfane sulfur in cells and in vivo. 


\section{Notes}

The authors declare no competing financial interest.

\section{Acknowledgment}

We thank the National Nature Science Foundation of China (No. 21405172, No. 21775162, No. 21575159, and No. 21522706), the program of Youth Innovation Promotion Association, CAS (Grant 2015170), and State Key Laboratory of Environmental Chemistry and Ecotoxicology, Research Center for Eco-Environmental Sciences, CAS (Grant KF2016-22).

\section{Appendix A. Supplementary data}

Supplementary data related to this article can be found at https://doi.org/10.1016/j.biomaterials.2018.01.011.

\section{References}

[1] M.G. Iciek, L. Wlodek, Biosynthesis and biological properties of compounds containing highly reactive, reduced sulfane sulfur, Pol. J. Pharmacol. 53 (2001) $215-225$.

[2] J.I. Toohey, A.J.L. Cooper, Thiosulfoxide (sulfane) sulfur: new chemistry and new regulatory roles in biology, Molecules 19 (2014) 12789-12813.

[3] M. Gao, F. Yu, H. Chen, L. Chen, Near-infrared fluorescent probe for imaging mitochondrial hydrogen polysulfides in living cells and in vivo, Anal. Chem. 87 (2015) 3631-3638.

[4] T. Ida, T. Sawa, H. Ihara, Y. Tsuchiya, Y. Watanabe, Y. Kumagai, M. Suematsu, H. Motohashi, S. Fujii, T. Matsunaga, M. Yamamoto, K. Ono, N.O. Devarie-Baez, M. Xian, J.M. Fukuto, T. Akaike, Reactive cysteine persulfides and S-polythiolation regulate oxidative stress and redox signaling, P. Natl. Acad. Sci. USA 111 (2014) 7606-7611.

[5] F. Yu, M. Gao, M. Li, L. Chen, A dual response near-infrared fluorescent probe for hydrogen polysulfides and superoxide anion detection in cells and in vivo Biomaterials 63 (2015) 93-101.

[6] H. Jurkowska, W. Placha, N. Nagahara, M. Wróbel, The expression and activity of cystathionine- $\gamma$-lyase and 3-mercaptopyruvate sulfurtransferase in human neoplastic cell lines, Amino Acids 41 (2011) 151-158.

[7] J.I. Toohey, Sulphane sulphur in biological systems: a possible regulatory role, Biochem. J. 264 (1989) 625

[8] B.D. Paul, S.H. Snyder, $\mathrm{H}_{2} \mathrm{~S}$ signalling through protein sulfhydration and beyond, Nat. Rev. Mol. Cell Biol. 13 (8) (2012) 499-507.

[9] L. Li, P. Rose, P.K. Moore, Hydrogen sulfide and cell signaling, Annu. Rev. Pharmacol. 51 (2011) 169-187.

[10] N. Shibuya, M. Tanaka, M. Yoshida, Y. Ogasawara, T. Togawa, K. Ishii, H. Kimura, 3-Mercaptopyruvate sulfurtransferase produces hydrogen sulfide and bound sulfane sulfur in the brain, Antioxid. Redox. Sign. 11 (2009) 703-714.

[11] H. Kimura, Physiological role of hydrogen sulfide and polysulfide in the central nervous system, Neurochem. Int. 63 (2013) 492-497.

[12] Y. Kimura, Y. Mikami, K. Osumi, M. Tsugane, J.I. Oka, H. Kimura, Polysulfides are possible H2S-derived signaling molecules in rat brain, Faseb. J. 27 (2013) 2451-2457.

[13] M. Kondoh, N. Ohga, K. Akiyama, Y. Hida, N. Maishi, A.M. Towfik, N. Inoue, M. Shindoh, K. Hida, Hypoxia-induced reactive oxygen species cause chromosomal abnormalities in endothelial cells in the tumor microenvironment, PLoS One 8 (11) (2013).

[14] J.M. Lluis, F. Buricchi, P. Chiarugi, A. Morales, J.C. Fernandez-Checa, Dual role of mitochondrial reactive oxygen species in hypoxia signaling: activation of nuclear factor-kappa B via c-SRC- and oxidant-dependent cell death, Cancer Res. 67 (2007) 7368-7377.

[15] R. Rathore, Y.M. Zheng, C.F. Niu, Q.H. Liu, A. Korde, Y.S. Ho, Y.X. Wang, Hypoxia activates NADPH oxidase to increase ROS (i) and $\mathrm{Ca}^{2+}$ (i) through the mito chondrial ROS-PKC epsilon signaling axis in pulmonary artery smooth muscle cells, Free Radical. Bio. Med. 45 (2008) 1223-1231.

[16] C. Yang, Z. Yang, M. Zhang, Q. Dong, X. Wang, A. Lan, F. Zeng, P. Chen, C. Wang J. Feng, Hydrogen sulfide protects against chemical hypoxia-induced cytotoxicity and inflammation in hacat cells through inhibition of ros/nf-kappa b/ cox-2 pathway, PLoS One 6 (2011).

[17] T.L. Clanton, Hypoxia-induced reactive oxygen species formation in skeleta muscle, J. Appl. Physiol. 102 (2007) 2379-2388.

[18] M. Valko, C.J. Rhodes, J. Moncol, M. Izakovic, M. Mazur, Free radicals, metals and antioxidants in oxidative stress-induced cancer, Chem. Biol. Interact. 160 (2006) $1-40$.

[19] E.E. Battin, J.L. Brumaghim, Antioxidant activity of sulfur and selenium: review of reactive oxygen species scavenging, glutathione peroxidase, and metal-binding antioxidant mechanisms, Cell Biochem. Biophys. 55 (2009) $1-23$.
[20] M. Li, L. Nie, Y. Hu, X. Yan, L. Xue, L. Chen, H. Zhou, Y. Zheng, Chronic intermittent hypoxia promotes expression of 3-mercaptopyruvate sulfurtransferase in adult rat medulla oblongata, Auton. Neurosci. Basic 179 (2013) 84-89.

[21] M. Fu, W.H. Zhang, L.Y. Wu, G.D. Yang, H.Z. Li, R. Wang, Hydrogen sulfide $\left(\mathrm{H}_{2} \mathrm{~S}\right)$ metabolism in mitochondria and its regulatory role in energy production, P. Natl. Acad. Sci. USA 109 (2012) 2943-2948.

[22] J.L. Wood, Sulfane sulfur, Meth. Enzymol. 143 (1987) 25-29.

[23] T. Togawa, M. Ogawa, M. Nawata, Y. Ogasawara, K. Kawanabe, S. Tanabe, High-performance liquid-chromatographic determination of bound sulfide and sulfite and thiosulfate at their low-levels in human serum by precolumn fluorescence derivatization with monobromobimane, Chem. Pharm. Bull. 40 (1992) 3000-3004.

[24] X. Chen, Y. Zhou, X. Peng J. Yoon, Fluorescent and colorimetric probes for detection of thiols, Chem. Soc. Rev. 39 (2010) 2120-2135.

[25] M. Gao, F. Yu, C. Lv, J. Choo, L. Chen, Fluorescent chemical probes for accurate tumor diagnosis and targeting therapy, Chem. Soc. Rev, 46 (2017) 2237-2271.

[26] Y. Yang, Q. Zhao, W. Feng, F. Li, Luminescent chemodosimeters for bioimaging, Chem. Rev. 113 (2012) 192-270.

[27] L. Yuan, W. Lin, K. Zheng, L. He, W. Huang, Far-red to near infrared analyteresponsive fluorescent probes based on organic fluorophore platforms for fluorescence imaging, Chem. Soc. Rev. 42 (2013) 622-661.

[28] J. Fan, M. Hu, P. Zhan, X. Peng, Energy transfer cassettes based on organic fluorophores: construction and applications in ratiometric sensing, Chem. Soc. Rev. 42 (2013) 29-43.

[29] X. Li, X. Gao, W. Shi, H. Ma, Design strategies for water-soluble small molecular chromogenic and fluorogenic probes, Chem. Rev. 114 (2013) 590-659.

[30] W. Chen, C. Liu, B. Peng, Y. Zhao, A. Pacheco, M. Xian, New fluorescent probes for sulfane sulfurs and the application in bioimaging, Chem. Sci. 4 (2013) 2892-2896.

[31] C.M. Park, L. Weerasinghe, JJ. Day, J.M. Fukuto, M. Xian, Persulfides: current knowledge and challenges in chemistry and chemical biology, Mol. Biosyst. 11 (2015) 1775-1785

[32] Y. Takano, H. Echizen, K. Hanaoka, Fluorescent probes and selective inhibitors for biological studies of hydrogen sulfide- and polysulfide-mediated signaling, Antioxid. Redox. Sign. 27 (2017) 669-683.

[33] V.S. Lin, W. Chen, M. Xian, C.J. Chang, Chemical probes for molecular imaging and detection of hydrogen sulfide and reactive sulfur species in biological systems, Chem. Soc. Rev. 44 (2015) 4596-4618.

[34] F. Yu, X. Han, L. Chen, Fluorescent probes for hydrogen sulfide detection and bioimaging, Chem. Commun. 50 (2014) 12234-12249.

[35] W. Chen, E.W. Rosser, T. Matsunaga, A. Pacheco, T. Akaike, M. Xian, The development of fluorescent probes for visualizing intracellular hydrogen polysulfides, Angew. Chem. Int. Ed. 54 (2015) 13961-13965.

[36] M. Gao, R. Wang, F. Yu, J. You, L. Chen, A near-infrared fluorescent probe for the detection of hydrogen polysulfides biosynthetic pathways in living cells and in vivo, Analyst 140 (2015) 3766-3772.

[37] Q. Han, Z. Mou, H. Wang, X. Tang, Z. Dong, L. Wang, X. Dong, W. Liu, Highly selective and sensitive one- and two-photon ratiometric fluorescent probe for intracellular hydrogen polysulfide sensing, Anal. Chem. 88 (2016) 7206-7212.

[38] H. Shang, H. Chen, Y. Tang, R. Guo, W. Lin, Construction of a two-photon fluorescent turn-on probe for hydrogen persulfide and polysulfide and its bioimaging application in living mice, Sensor Actuat. B Chem. 230 (2016) $773-778$.

[39] W. Chen, E.W. Rosser, D. Zhang, W. Shi, Y. Li, W.-J. Dong, H. Ma, D. Hu, M. Xian, A specific nucleophilic ring-opening reaction of aziridines as a unique platform for the construction of hydrogen polysulfides sensors, Org. Lett. 17 (2015) 2776-2779

[40] Y. Huang, F. Yu, J. Wang, L. Chen, Near-Infrared Fluorescence probe for in situ detection of superoxide anion and hydrogen polysulfides in mitochondrial oxidative stress, Anal. Chem. 88 (2016) 4122-4129.

[41] X. Han, F. Yu, X. Song, L. Chen, Quantification of cysteine hydropersulfide with a ratiometric near-infrared fluorescent probe based on selenium-sulfur exchange reaction, Chem. Sci. 7 (2016) 5098-5107.

[42] M. Iciek, L. Wlodek, Biosynthesis and biological properties of compounds containing highly reactive, reduced sulfane sulfur, Pol. J. Pharmacol. 53 (2001) $215-225$.

[43] R. Greiner, Z. Palinkas, K. Baesell, D. Becher, H. Antelmann, P. Nagy, T.P. Dick, Polysulfides link $\mathrm{h}_{2} \mathrm{~S}$ to protein thiol oxidation, Antioxid. Redox. Sign. 19 (2013), 1749-U122.

[44] K. Ono, T. Akaike, T. Sawa, Y. Kumagai, D.A. Wink, D.J. Tantillo, A.J. Hobbs, P. Nagy, M. Xian, J. Lin, J.M. Fukuto, Redox chemistry and chemical biology of $\mathrm{H}_{2} \mathrm{~S}$, hydropersulfides, and derived species: implications of their possible biological activity and utility, Free Radical. Bio. Med. 77 (2014) 82-94.

[45] M. Iciek, D. Kowalczyk-Pachel, A. Bilska-Wilkosz, I. Kwiecien, M. Gorny, L. Wlodek, S-sulfhydration as a cellular redox regulation, Biosci. Rep. 36 (2) (2015).

[46] J. Toohey, Sulfur signaling: is the agent sulfide or sulfane? Anal. Biochem. 413 (2011) 1-7.

[47] M.P. Murphy, How mitochondria produce reactive oxygen species, Biochem. J. 417 (2009) 1-13.

[48] M. Fu, W. Zhang, L. Wu, G. Yang, H. Li, R. Wang, Hydrogen sulfide $\left(\mathrm{H}_{2} \mathrm{~S}\right)$ metabolism in mitochondria and its regulatory role in energy production, P. Natl. Acad. Sci. USA 109 (2012) 2943-2948.

[49] K. Kundu, S.F. Knight, N. Willett, S. Lee, W.R. Taylor, N. Murthy, Hydrocyanines: a class of fluorescent sensors that can image reactive oxygen species 
in cell culture, tissue, and in vivo, Angew. Chem. Int. Ed. 48 (2009) 299-303.

[50] W. Piao, S. Tsuda, Y. Tanaka, S. Maeda, F. Liu, S. Takahashi, Y. Kushida, T. Komatsu, T. Ueno, T. Terai, Development of azo-based fluorescent probes to detect different levels of hypoxia, Angew. Chem. Int. Ed. 52 (2013) $13028-13032$

[51] S. Takahashi, W. Piao, Y. Matsumura, T. Komatsu, T. Ueno, T. Terai, T. Kamachi, M. Kohno, T. Nagano, K. Hanaoka, Reversible off-on fluorescence probe for hypoxia and imaging of hypoxia-normoxia cycles in live cells, J. Am. Chem. Soc. 134 (2012) 19588-19591.

[52] E. Takahashi, M. Sato, Imaging of oxygen gradients in monolayer cultured cells using green fluorescent protein, Am. J. Physiol Cell. Ph 299 (2010) C1318-C1323.

[53] G.L. Semenza, Angiogenesis ischemic and neoplastic disorders, Annu. Rev. Med. 54 (2003) 17-28.

[54] Z. Huang, S. Shiva, D.B. Kim-Shapiro, R.P. Patel, L.A. Ringwood, C.E. Irby, K.T. Huang, C. Ho, N. Hogg, A.N. Schechter, Enzymatic function of hemoglobin as a nitrite reductase that produces NO under allosteric control, J. Clin. Invest.
115 (2005) 2099.

[55] J.H. Crawford, T.S. Isbell, Z. Huang, S. Shiva, B.K. Chacko, A.N. Schechter, V.M. Darley-Usmar, J.D. Kerby, J.D. Lang, D. Kraus, Hypoxia, red blood cells, and nitrite regulate NO-dependent hypoxic vasodilation, Blood 107 (2006) 566-574.

[56] C. Murdoch, M. Muthana, C.E. Lewis, Hypoxia regulates macrophage functions in inflammation, J. Immunol. 175 (2005) 6257-6263.

[57] S. Kizaka-Kondoh, M. Inoue, H. Harada, M. Hiraoka, Tumor hypoxia: a target for selective cancer therapy, Cancer Sci. 94 (2003) 1021-1028.

[58] A.L. Harris, Hypoxia-a key regulatory factor in tumour growth, Nat. Rev. Cancer 2 (2002) 38-47.

[59] G. Mugesh, H.B. Singh, Synthetic organoselenium compounds as antioxidants: glutathione peroxidase activity, Chem. Soc. Rev. 29 (2000) 347-357.

[60] V.S. Lin, A.R. Lippert, C.J. Chang, Cell-trappable fluorescent probes for endogenous hydrogen sulfide signaling and imaging $\mathrm{H}_{2} \mathrm{O}_{2}$-dependent $\mathrm{H}_{2} \mathrm{~S}$ production, P. Natl. Acad. Sci. USA 110 (2013) 7131-7135. 